\title{
Drug-drug interactions and adverse drug reactions in polypharmacy among older adults: an integrative review ${ }^{1}$
}

\author{
Maria Cristina Soares Rodrigues² \\ Cesar de Oliveira ${ }^{3}$
}

\begin{abstract}
Objective: to identify and summarize studies examining both drug-drug interactions (DDI) and adverse drug reactions (ADR) in older adults polymedicated. Methods: an integrative review of studies published from January 2008 to December 2013, according to inclusion and exclusion criteria, in MEDLINE and EMBASE electronic databases were performed. Results: forty-seven fulltext studies including 14,624,492 older adults ( $\geq 60$ years) were analyzed: $24(51.1 \%)$ concerning ADR, 14 (29.8\%) DDI, and 9 studies (19.1\%) investigating both DDI and ADR. We found a variety of methodological designs. The reviewed studies reinforced that polypharmacy is a multifactorial process, and predictors and inappropriate prescribing are associated with negative health outcomes, as increasing the frequency and types of ADRs and DDIs involving different drug classes, moreover, some studies show the most successful interventions to optimize prescribing. Conclusions: DDI and ADR among older adults continue to be a significant issue in the worldwide. The findings from the studies included in this integrative review, added to the previous reviews, can contribute to the improvement of advanced practices in geriatric nursing, to promote the safety of older patients in polypharmacy. However, more research is needed to elucidate gaps.
\end{abstract}

Descriptors: Aged; Polypharmacy; Evidence-Based Practice; Review.

\footnotetext{
${ }^{1}$ Supported by Coordenação de Aperfeiçoamento de Pessoal de Nível Superior (CAPES), Brazil, process \# BEX 4259/13-0.

2 PhD, Associate Professor, Departamento de Enfermagem, Faculdade de Ciências da Saúde, Universidade de Brasília, Brasília, DF, Brazil. Scholarship holder from Coordenação de Aperfeiçoamento de Pessoal de Nível Superior (CAPES), Brazil.

${ }^{3}$ Researcher, Departament Epidemiology and Public Health, University College London, London, United Kingdom.
}

How to cite this article

Rodrigues MCS, Oliveira C. Drug-drug interactions and adverse drug reactions in polypharmacy among older adults: an integrative review. Rev. Latino-Am. Enfermagem. 2016;24:e2800. [Access in: DOI: http://dx.doi.org/10.1590/1518-8345.1316.2800. ]; Available 


\section{Introduction}

The world is on the brink of a demographic milestone. In about five years' time, the number of people aged 65 or older will outnumber children under age 5 . Driven by falling fertility rates and remarkable increases in life expectancy, population ageing will continue, even accelerate. The number of people aged 65 or older is projected to grow from an estimated 524 million in 2010 to nearly 1.5 billion in 2050, with most of the increase in developing countries ${ }^{(1)}$.

Ageing, one of the most complex biological phenomena, is a multifaceted process in which several physiological changes occur at both the tissue and the whole-organism level, occurring in cascade, especially post-reproduction ${ }^{(2)}$. The changes characterized by ageing include: changes in biochemical composition of tissues; progressive decrease in physiological capacity; reduced ability to adapt to stimuli; increased susceptibility and vulnerability to disease and increased risk of death ${ }^{(3)}$.

Age related chronic diseases such as dyslipidemia, hypertension, diabetes, and depression usually require the use of multiple drugs, a state known as polypharmacy. This refers to the use of multiple medications and/ or more medications than clinically indicated. It is estimated that more than $40 \%$ of adults aged 65 or older use 5 or more medications, and $12 \%$ use 10 or more different medications(4). However, the magnitude of the problem among older adults is still scarcely known in most countries.

It is well known in the literature that polypharmacy increases the use of inappropriate drugs, leading to the underuse of essential medicines for the appropriate control of conditions prevalent in the older adults. In addition, it sets up a barrier to treatment adherence in that it creates complex therapeutic regimens, and enables the occurrence of medication errors, drug-drug interactions, adverse reactions, and poor quality of life. It increases morbidity, mortality, and complexity of care. It also imposes a huge financial burden on both the older adults and health system ${ }^{(5)}$.

Furthermore, attention should be paid to the fact that the body of the older adults presents changes in their physiological functions that may lead to a differentiated pharmacokinetics and greater sensitivity to both therapeutic and adverse drug effects ${ }^{(5)}$. Pharmacokinetics, pharmacodynamics, and clinical outcomes are affected by a number of patient-specific factors, including age, sex, ethnicity, genetics, disease processes, polypharmacy, drug dose and frequency, social factors, and many other factors ${ }^{(6)}$.
The scenario above highlights that population ageing is a global phenomenon and the practice of polypharmacy is dangerous for patients, in particular for older adults, because favors the emergence of drugdrug interactions (DDI), adverse drug reactions (ADR), side effects, longer hospital stays, iatrogenic disease and may also lead to complications that induce the patient's death. Thus, the purpose of the present study was to conduct a broader integrative review aimed at identifying and summarizing studies examining both DDIs and ADRs in older adults polymedicated.

\section{Methods}

The stages of this integrative review include: problem identification, formulating the appropriate question to be investigated; literature search with selection of articles according to predetermined criteria; data evaluation extracting data from each study summary of results; data analysis and presentation of results( ${ }^{(7)}$. The following describes the steps of the integrative review for this study.

To elaborate the guiding question was applied to the PICO strategy defining population "older adults", intervention "use of multiple medications/polypharmacy" and outcome "occurrence of drug interactions and adverse drug reactions". Thus, the central question of this integrative review was: What is the scientific evidence available, demonstrating the occurrence of drug-drug interactions and adverse drug reactions in older (i.e. $\geq 60$ years of age) polymedicated adults?

For the selection of articles, studies published in the English, Spanish and Portuguese languages in the period between January 2008 and December 2013 were eligible. The time period was based on the existence of two previous literature reviews. One in which investigated observational studies examining the epidemiology of polypharmacy, reviewing randomized controlled studies that have been published in the past 2 decades (from 1986 until June 2007) designed to reduce polypharmacy in older adults ${ }^{(8)}$; and, another review who reviewed the body of literature addressing polypharmacy in individuals aged 60 years and older, between January 1991 and October 2003, to (a) determine primary care providers' definition of polypharmacy, (b) explore how polypharmacy was assessed in primary care, and (c) seek tested interventions that address polypharmacy ${ }^{(9)}$.

Additionally, studies obtained from primary sources, represented by original scientific articles, surveys that have shown data on the occurrence of DDI and ADR in older adults ( $\geq 60$ years of age), female and male sex, were on multiple medications (polypharmacy) were selected. The following were excluded from this review: 
articles on drug-disease, drug-food, drug-alcohol interactions and drug-nutritional status, abstracts, case study articles, news, commentary, reflection, systematic review articles, clinical updates, expert opinion, studies concomitantly involving child (birth - 18 years), adults ( $\geq 19$ years), middle aged ( $\geq 45$ years) and older people ( $\geq 60$ years), research with qualitative approach, editorials, consensus, study protocols, clinical guidelines, reviews, short communication, monographs, theoretical studies and economic evaluation studies and articles published before 2008 .

The following bibliographic databases were researched: International Medical Literature Analysis and Retrieval System Online (MEDLINE) via PubMed and EMBASE. In these databases were used Medical Subject Headings Terms (MeSH) and Emtree terms. The main descriptors adopted in the search strategy for primary studies were: older adults, polypharmacy, drug interactions, adverse drug reactions and aged, combined using the Boolean operators AND and OR.

After searched, all articles were screened by reading their title, abstract and, when necessary, the content briefly, and, thereby, identifying those papers potentially addressing the topic. The selected articles were analyzed initially and in a second stage, they were read in more detail regarding their content. Finally, the selected articles had their data synthesized. To summarize the data of the selected articles and aiming to ensure that all relevant information was extracted, we applied to each study a validated instrument by Ganong(10).

In order to determine the relevance of articles captured in the searched databases, two examiners performed the synthesis of the data of interest independently which was followed by the thematic analysis of the papers. Each item synthesized/recorded

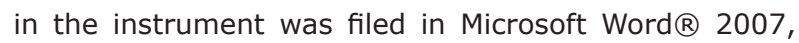
generating a database. All disagreements were resolved by discussion.

The results and data analysis are presented in descriptive form.

\section{Results}

A total of 409 references were identified and fortyseven were included in the final analysis. For details, see the flow diagram (Figure 1).

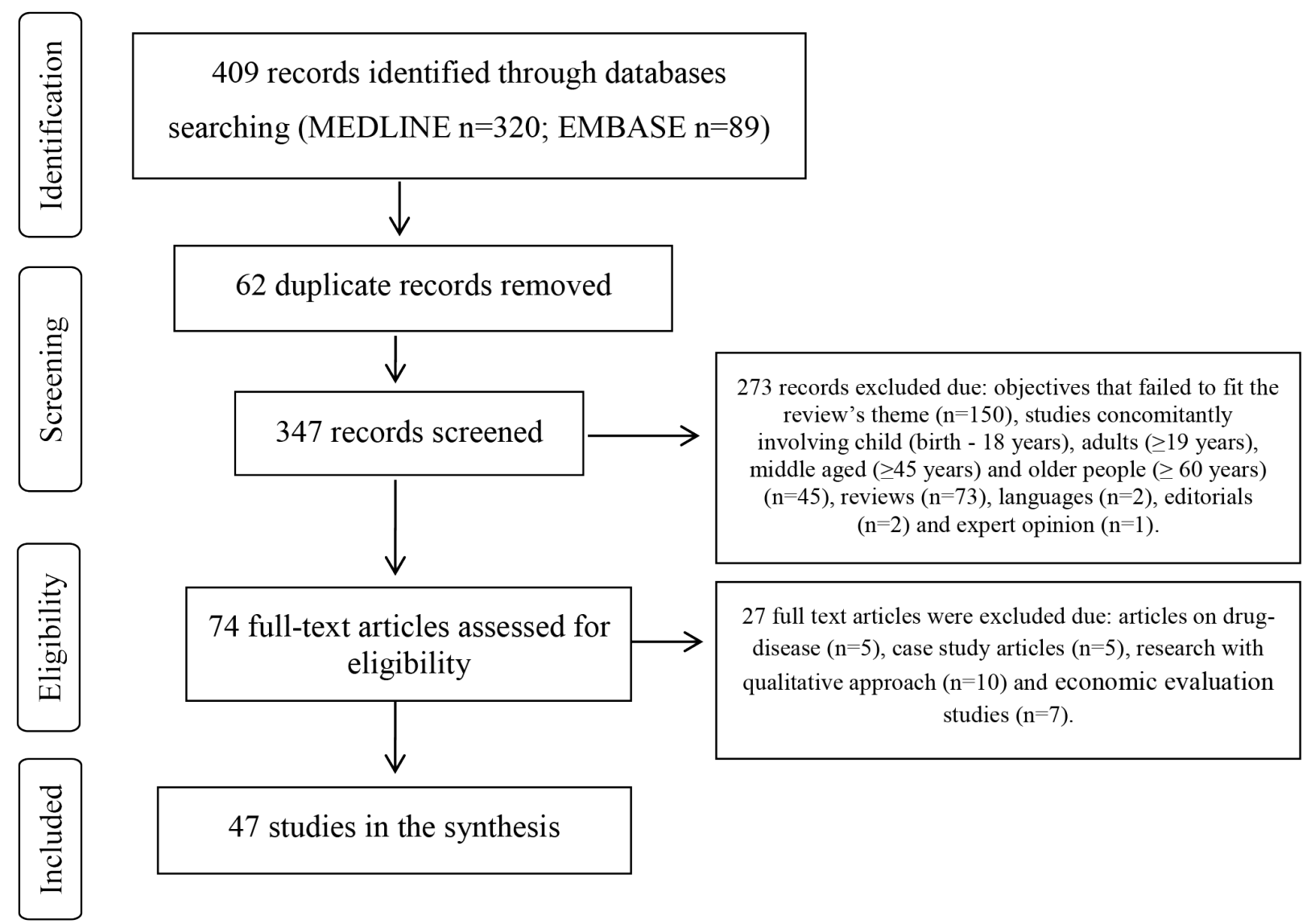

Figure 1 - Flow diagram of the articles screened, assessed for eligibility, included and excluded. London, United Kingdom, 2013 
The articles analyzed were from different countries: 9 from (19.1\%) the United States, 7 (14.9\%) from Canada, 6 (12.8\%) from Brazil, 4 (8.5\%) from Ireland, 3 (6.4\%, respectively) from Belgium and India, 2 (4.3\%, respectively) from Australia, Croatia, Spain, Norway and 1 (2.1\%, respectively) from France, Indonesia, Netherlands, Singapore, Switzerland, Sweden, Taiwan. Regarding language, 46 (97.9\%) articles were written in English and only one (2.1\%) in Spanish. Descriptive and analytical studies have been included: cross-sectional
( $n=9 ; 19.1 \%)$, followed by cohort studies $(n=7 ; 14.9 \%)$, nested case-control $(n=6 ; 12.8 \%)$ and prospective and prospective observational ( $n=5 ; 10.6 \%$, respectively), among others. Considering all the 47 articles examined, a total of $14,624,492$ older adults/patients ( $\geq 60$ years) were included, predominantly of hospitals ( $n=22$ articles; $45.8 \%)$. Concerning the authorship of publications, 31 $(66.0 \%)$ are of multidisciplinary researchers, 8 (17.0\%) from pharmacists, 7 (14.9\%) from physicians and 1 (2.1\%) from dentists.

\begin{tabular}{|c|c|c|c|c|}
\hline Source & Setting & Design & Aim & $\begin{array}{c}\text { Sample } \\
\left.\text { Median age (IQR* or } \text { SD }^{\dagger}\right)\end{array}$ \\
\hline $\begin{array}{l}\text { Gallagher and } \\
\text { O'Mohony } \\
\text { Ireland, 2008(11) }\end{array}$ & $\begin{array}{l}\text { University teaching } \\
\text { hospital }\end{array}$ & Prospective study & $\begin{array}{l}\text { Compare the performance of } \\
\text { Screening Tool of Older Persons' } \\
\text { Potentially Inappropriate Prescriptions } \\
\text { to that Beers' criteria in detecting } \\
\text { potentially inappropriate medicines } \\
\text { and related ADR in older patients } \\
\text { presenting for hospital admissions. }\end{array}$ & $\begin{array}{l}\mathrm{n}=715 \\
77 \text { years } \\
\text { (IQR: } 72-82)\end{array}$ \\
\hline $\begin{array}{l}\text { Gallagher } \\
\text { et al. } \\
\text { Ireland, 2008 } \\
\end{array}$ & $\begin{array}{l}\text { University teaching } \\
\text { hospital }\end{array}$ & $\begin{array}{l}\text { Prospective, } \\
\text { observational study }\end{array}$ & $\begin{array}{l}\text { Determine the prevalence of } \\
\text { potentially inappropriate prescriptions } \\
\text { (using Beers' criteria) in an unselected } \\
\text { community-dwelling population } \\
\text { aged } 65 \text { years and over, requiring } \\
\text { admission to an acute general } \\
\text { hospital. }\end{array}$ & $\begin{array}{l}\mathrm{n}=597 \\
77 \text { years (SD: } 7)\end{array}$ \\
\hline $\begin{array}{l}\text { Zhang et al. } \\
\text { Australia, 2009(13) }\end{array}$ & $\begin{array}{l}\text { All public and } \\
\text { private hospitals in } \\
\text { Western } \\
\text { Australia }\end{array}$ & $\begin{array}{l}\text { Population based } \\
\text { retrospective } \\
\text { cohort study }\end{array}$ & $\begin{array}{l}\text { Identify factors that predict repeat } \\
\text { admission to hospital for ADR in older } \\
\text { adults. }\end{array}$ & $\begin{array}{l}n=28,548 \\
\text { residents aged } \geq 60 \text { years }\end{array}$ \\
\hline $\begin{array}{l}\text { Juurlink } \\
\text { et al. } \\
\text { Canada, 2009(14) }\end{array}$ & $\begin{array}{l}\text { Ontario residents } \\
\text { who were } \\
\text { discharged from } \\
\text { hospital between } \\
\text { Apr. 1, 2002, and } \\
\text { Dec. } 31,2007\end{array}$ & $\begin{array}{l}\text { Population-based } \\
\text { nested } \\
\text { case-control study }\end{array}$ & $\begin{array}{l}\text { Characterize whether the concomitant } \\
\text { use of a proton pump inhibitor with } \\
\text { clopidogrel was associated with } \\
\text { adverse outcomes among older } \\
\text { patients discharged from hospital after } \\
\text { acute myocardial infarction. }\end{array}$ & $\begin{array}{l}\mathrm{n}=734 \text { cases } \\
\mathrm{n}=2,057 \text { controls } \\
77 \text { years } \\
\text { (IQR: } 72-83)\end{array}$ \\
\hline $\begin{array}{l}\text { Rassen et al. } \\
\text { U.S, 2009(15) }\end{array}$ & $\begin{array}{l}\text { Data were } \\
\text { collected from } \\
\text { patients enrolled } \\
\text { in } 3 \text { large health } \\
\text { insurance } \\
\text { Programs in } \\
\text { British Columbia, } \\
\text { Pennsylvania and } \\
\text { New Jersey }\end{array}$ & $\begin{array}{l}\text { Retrospective } \\
\text { study }\end{array}$ & $\begin{array}{l}\text { Examine adverse outcomes in } \\
\text { patients taking clopidogrel who were } \\
\text { treated concurrently with proton pump } \\
\text { inhibitors vs those taking clopidogrel } \\
\text { alone. The outcomes studied were } \\
\text { myocardial infarction hospitalization, } \\
\text { revascularization, and death. }\end{array}$ & $\begin{array}{l}\mathrm{n}=18,565 \text { (total) } \\
\text { British Columbia } \\
74 \text { years } \\
\text { (range: } 65-100 \text { ) } \\
\text { Pennsylvania } \\
78 \text { years } \\
\text { (range: } 65-104 \text { ) } \\
\text { New Jersey } \\
78 \text { years } \\
\text { (range: } 65-101 \text { ) }\end{array}$ \\
\hline $\begin{array}{l}\text { Miquel et al. } \\
\text { Spain, 2010(16) }\end{array}$ & $\begin{array}{l}\text { Three health care } \\
\text { settings in Madrid: } \\
\text { a hospital geriatric } \\
\text { assessment } \\
\text { outpatient clinic, a } \\
\text { primary care clinic } \\
\text { of the National } \\
\text { Health Service, and } \\
\text { an assisted private } \\
\text { nursing home }\end{array}$ & $\begin{array}{l}\text { Cross-sectional } \\
\text { study }\end{array}$ & $\begin{array}{l}\text { Compare the performance of two } \\
\text { different tools, i.e. Beers' criteria } \\
\text { and Screening Tool of Older } \\
\text { Persons' Potentially Inappropriate } \\
\text { Prescriptions/ Screening Tool to Alert } \\
\text { doctors to the Right, i.e. appropriate, } \\
\text { indicated treatment in the detection } \\
\text { of potentially inappropriate drugs and } \\
\text { prescribing omissions of appropriate } \\
\text { drugs in older adults cared in the } \\
\text { three different settings. }\end{array}$ & $\begin{array}{l}n=150 \\
81.6(S D: 6.3) \\
\text { Primary care } \\
(n=50 ; 78.8 \pm 5.3) \\
\text { Geriatric clinic } \\
(n=50 ; 81.5 \pm 7.6) \\
\text { Nursing home } \\
(n=50 ; 84.5 \pm 7.6)\end{array}$ \\
\hline $\begin{array}{l}\text { Weir et al. } \\
\text { Canada, 2010(17) }\end{array}$ & $\begin{array}{l}\text { Residents of } \\
\text { Ontario }\end{array}$ & $\begin{array}{l}\text { Two nested case- } \\
\text { control studies }\end{array}$ & $\begin{array}{l}\text { Examine the association between } \\
\text { hospitalization for hyperkalemia } \\
\text { and the use of trimethoprim/ } \\
\text { sulfamethoxazole antibiotics in older } \\
\text { adults receiving } \beta \text {-blockers. }\end{array}$ & $\begin{array}{l}n=189 \text { cases } \\
n=641 \text { controls } \\
80 \text { years } \\
\text { (73-84 cases) } \\
\text { ( } 74-84 \text { controls) }\end{array}$ \\
\hline
\end{tabular}




\begin{tabular}{|c|c|c|c|c|}
\hline Source & Setting & Design & Aim & $\begin{array}{c}\text { Sample } \\
\text { Median age }\left(\mathrm{IQR}^{*} \text { or } \mathrm{SD}^{\dagger}\right)\end{array}$ \\
\hline $\begin{array}{l}\text { Kelly et al. } \\
\text { Canada, } 2010^{(18)}\end{array}$ & $\begin{array}{l}\text { Residents of } \\
\text { Ontario }\end{array}$ & $\begin{array}{l}\text { Population based } \\
\text { cohort study }\end{array}$ & $\begin{array}{l}\text { Characterize whether some } \\
\text { selective serotonin reuptake inhibitor } \\
\text { antidepressants reduce tamoxifen's } \\
\text { effectiveness by inhibiting its } \\
\text { bioactivation by cytochrome P450 } \\
\text { 2D6. }\end{array}$ & $\begin{array}{l}\mathrm{n}=7,489 \\
74 \text { years } \\
\text { (IQR: } 70-79)\end{array}$ \\
\hline $\begin{array}{l}\text { Somers et al. } \\
\text { Australia, } 2010^{(19)}\end{array}$ & $\begin{array}{l}\text { Residents with } \\
\text { dementia from } \\
\text { residential aged } \\
\text { care facilities }\end{array}$ & Uniformed & $\begin{array}{l}\text { Describe the patterns of prescribing } \\
\text { for a sample of older people with } \\
\text { dementia living in residential aged } \\
\text { care facilities, including: number of } \\
\text { medications prescribed; extent of } \\
\text { psycholeptic use; use of potentially } \\
\text { inappropriate medicines; drug-drug } \\
\text { interactions; anticholinergic burden. }\end{array}$ & $\begin{array}{l}\mathrm{n}=351 \\
85,24 \text { years } \\
\text { (SD: } 7,87)\end{array}$ \\
\hline $\begin{array}{l}\text { Harugeri } \\
\text { et al. } \\
\text { India, 2010(20) }\end{array}$ & $\begin{array}{l}\text { Medicine wards } \\
\text { at two teaching } \\
\text { hospitals }\end{array}$ & $\begin{array}{l}\text { Prospective } \\
\text { observational study }\end{array}$ & $\begin{array}{l}\text { Determine the prevalence and } \\
\text { predictors of potentially inappropriate } \\
\text { medicines use, and assess the } \\
\text { relationship between potentially } \\
\text { inappropriate medicines use and ADR } \\
\text { in the hospitalized older adults. }\end{array}$ & $\begin{array}{l}\mathrm{n}=814 \\
66 \text { years } \\
\text { (range: 60-95) }\end{array}$ \\
\hline $\begin{array}{l}\text { Bacic-Vrca } \\
\text { et al. } \\
\text { Croatia, 2010(21) }\end{array}$ & $\begin{array}{l}\text { Three community } \\
\text { pharmacies }\end{array}$ & Prospective study & $\begin{array}{l}\text { Assess the incidence and type of } \\
\text { potential, clinically significant DDI } \\
\text { in older outpatients with arterial } \\
\text { hypertension. }\end{array}$ & $\begin{array}{l}\mathrm{n}=265 \\
73 \text { years } \\
\text { (range: } 65-95 \text { ) }\end{array}$ \\
\hline $\begin{array}{l}\text { Rahmawati et al. } \\
\text { Indonesia, } 2010^{(22)}\end{array}$ & $\begin{array}{l}\text { A private hospital at } \\
\text { Yogyakarta }\end{array}$ & $\begin{array}{l}\text { Descriptive } \\
\text { observational } \\
\text { retrospective study }\end{array}$ & $\begin{array}{l}\text { Determine the occurrence of DDI } \\
\text { and the association between the } \\
\text { numbers of drugs used per day during } \\
\text { hospitalization with the number of } \\
\text { potential DDIs. }\end{array}$ & $\begin{array}{l}n=100 \\
75.1 \text { years } \\
\text { (SD: } 5.8)\end{array}$ \\
\hline $\begin{array}{l}\text { Wright et al. } \\
\text { Canada, 2011(23) }\end{array}$ & $\begin{array}{l}\text { Residents of } \\
\text { Ontario }\end{array}$ & $\begin{array}{l}\text { Population-based, } \\
\text { nested, case- } \\
\text { crossover study }\end{array}$ & $\begin{array}{l}\text { Characterize the clinical } \\
\text { consequences of macrolide use } \\
\text { among patients who were taking a } \\
\text { calcium-channel blocker. }\end{array}$ & $\begin{array}{l}\mathrm{n}=7,100 \\
77 \text { years } \\
\text { (IQR: } 72-83 \text { ) }\end{array}$ \\
\hline $\begin{array}{l}\text { Steinman } \\
\text { et al. } \\
\text { U.S, 2011(24) }\end{array}$ & $\begin{array}{l}\text { Veterans Affairs } \\
\text { Medical Center }\end{array}$ & $\begin{array}{l}\text { Prospective cohort } \\
\text { study }\end{array}$ & $\begin{array}{l}\text { Determine whether geriatric } \\
\text { conditions and functional impairment } \\
\text { are independent risk factors for ADRs. }\end{array}$ & $\begin{array}{l}\mathrm{n}=377 \\
74.2 \text { years } \\
(\mathrm{SD}: 5.5)\end{array}$ \\
\hline $\begin{array}{l}\text { Steinman } \\
\text { et al. } \\
\text { U.S, 2011(25) }\end{array}$ & $\begin{array}{l}\text { Patients admitted } \\
\text { to medical or } \\
\text { surgical wards at } \\
11 \text { Veterans Affairs } \\
\text { Medical Center }\end{array}$ & Uniformed & $\begin{array}{l}\text { Test the association between geriatric } \\
\text { conditions and ADR using data on } \\
\text { vulnerable elderly outpatients from the } \\
\text { Veterans Affairs Geriatric Evaluation } \\
\text { and Management (GEM) Drug Study. }\end{array}$ & $\begin{array}{l}n=808 \\
74 \text { years } \\
\text { (SD: } 6)\end{array}$ \\
\hline $\begin{array}{l}\text { Santos et al. } \\
\text { Brazil, 2011(26) }\end{array}$ & $\begin{array}{l}\text { Hospital Care } \\
\text { Foundation of } \\
\text { Paraiba, Campina } \\
\text { Grande }\end{array}$ & $\begin{array}{l}\text { Cross-sectional } \\
\text { descriptive study }\end{array}$ & $\begin{array}{l}\text { Study the consumption of drugs, } \\
\text { noting the possible ADR, DDI and the } \\
\text { presence of high-risk drugs prescribed } \\
\text { to older patient hospitalized. }\end{array}$ & $\begin{array}{l}n=65 \\
72.47 \text { years } \\
\text { (SD: } 8.37)\end{array}$ \\
\hline $\begin{array}{l}\text { Mendes-Netto } \\
\text { et al. } \\
\text { Brazil, 2011(27) }\end{array}$ & $\begin{array}{l}\text { Family Health Care } \\
\text { Unit in Aracaju, } \\
\text { Brazil. }\end{array}$ & $\begin{array}{l}\text { Cross-sectional } \\
\text { pilot study }\end{array}$ & $\begin{array}{l}\text { Assess drug-drug interactions in older } \\
\text { adults. }\end{array}$ & $\begin{array}{l}\mathrm{n}=34 \text { ( } 28 \text { women) } \\
\text { Subjects in age range } \\
60-84(48.57 \%) ; 60 \text { to } 69 \\
(40 \%) ; 70 \text { to } 79(79 \%), \\
\text { and } 80 \text { to } 84(11.42 \%) .\end{array}$ \\
\hline $\begin{array}{l}\text { Antoniou } \\
\text { et al. } \\
\text { Canada, 2011(28) }\end{array}$ & $\begin{array}{l}\text { Residents of } \\
\text { Ontario }\end{array}$ & $\begin{array}{l}\text { Population based } \\
\text { nested case-control } \\
\text { study }\end{array}$ & $\begin{array}{l}\text { Characterize the risk of admission } \\
\text { to hospital for hyperkalemia in older } \\
\text { patients treated with trimethoprim/ } \\
\text { sulfamethoxazole in combination with } \\
\text { spironolactone. }\end{array}$ & $\begin{array}{l}\mathrm{n}=1,031 \\
\text { ( } 248 \text { cases and } 783 \\
\text { controls) } \\
\text { Cases: } 82 \text { years } \\
\text { (IQR: } 75-87 \text { ) } \\
\text { Controls: } 81 \text { years } \\
\text { (IQR: } 75-87 \text { ) }\end{array}$ \\
\hline
\end{tabular}




\begin{tabular}{|c|c|c|c|c|}
\hline Source & Setting & Design & Aim & $\begin{array}{c}\text { Sample } \\
\text { Median age }\left(\text { (IQR }^{*} \text { or } \mathrm{SD}^{\dagger}\right)\end{array}$ \\
\hline $\begin{array}{l}\text { Varallo et al. } \\
\text { Brazil, 2011(29) }\end{array}$ & $\begin{array}{l}\text { Public teaching } \\
\text { hospital, internal } \\
\text { medicine ward }\end{array}$ & $\begin{array}{l}\text { Descriptive and } \\
\text { cross-sectional } \\
\text { study }\end{array}$ & $\begin{array}{l}\text { Estimate the prevalence of older } \\
\text { adults who had used potentially } \\
\text { inappropriate medicines before being } \\
\text { admitted to hospital and to identify the } \\
\text { risk factors and the hospitalizations } \\
\text { related to ADR arising from potentially } \\
\text { inappropriate medicines. }\end{array}$ & $\begin{array}{l}\mathrm{n}=60 \\
\text { Patients } \geq 60 \text { years }\end{array}$ \\
\hline $\begin{array}{l}\text { Gallagher } \\
\text { et al. } \\
\text { Ireland, 2011(30) }\end{array}$ & $\begin{array}{l}\text { Cork University } \\
\text { Hospital, an } 800- \\
\text { bed, state-funded, } \\
\text { tertiary medical } \\
\text { center in southern } \\
\text { Ireland serving an } \\
\text { urban and rural } \\
\text { population. }\end{array}$ & $\begin{array}{l}\text { Randomized } \\
\text { controlled study }\end{array}$ & $\begin{array}{l}\text { Test the hypothesis that screening } \\
\text { of hospitalized older patients' } \\
\text { medications against Screening } \\
\text { Tool of Older Persons' Potentially } \\
\text { Inappropriate Prescriptions/ Screening } \\
\text { Tool to Alert doctors to the Right, i.e. } \\
\text { appropriate, indicated Treatment, and } \\
\text { providing resultant recommendations } \\
\text { to the attending medical team, would } \\
\text { lead to significant and sustained } \\
\text { improvements in prescribing } \\
\text { appropriateness as compared with } \\
\text { usual hospital pharmaceutical care. }\end{array}$ & $\begin{array}{l}\mathrm{n}=382 \\
\text { (192 controls and } 190 \\
\text { intervention) } \\
\text { Control: } 77 \text { years (IQR:71- } \\
81.75) \\
\text { Intervention: } 74.5 \\
\text { (IQR:71-80) }\end{array}$ \\
\hline $\begin{array}{l}\text { Hanlon et al. } \\
\text { U.S, 2011(31) }\end{array}$ & $\begin{array}{l}133 \text { Veterans } \\
\text { Affairs Community } \\
\text { Living Center } \\
\text { patients. }\end{array}$ & Longitudinal study & $\begin{array}{l}\text { Examine prevalence and patient/site } \\
\text { level factors associated with potential } \\
\text { underuse, overuse and inappropriate } \\
\text { use of antidepressants. }\end{array}$ & $\begin{array}{l}n=3,692 \\
65 \text { years or older }\end{array}$ \\
\hline $\begin{array}{l}\text { Skaar and } \\
\text { O'Connor } \\
\text { US, 2011(32) }\end{array}$ & $\begin{array}{l}\text { Community- } \\
\text { dwelling Medicare } \\
\text { beneficiaries with a } \\
\text { dental visit in } 2006\end{array}$ & $\begin{array}{l}\text { The Medicare } \\
\text { Current Beneficiary } \\
\text { Survey }\end{array}$ & $\begin{array}{l}\text { Identify prescription drugs with the } \\
\text { potential for serious interactions and } \\
\text { estimate prevalent use among older } \\
\text { adults visiting the dentist. }\end{array}$ & $\begin{array}{l}n=14,361,198 \text { (estimated) } \\
\geq 65 \text { years } \\
\text { of age or older }\end{array}$ \\
\hline $\begin{array}{l}\text { Venturini } \\
\text { et al. } \\
\text { Brazil, 2011(33) }\end{array}$ & $\begin{array}{l}\text { Institute of Geriatric } \\
\text { and Gerontology } \\
\text { of Pontifícia } \\
\text { Universidade } \\
\text { Católica do Rio } \\
\text { Grande do Sul }\end{array}$ & $\begin{array}{l}\text { Observational } \\
\text { cross-sectional } \\
\text { study }\end{array}$ & $\begin{array}{l}\text { Analyze pharmacological interactions } \\
\text { among drugs taken by elderly patients } \\
\text { and their age and gender differences } \\
\text { in a population from Porto Alegre, } \\
\text { Brazil. }\end{array}$ & $\begin{array}{l}\mathrm{n}=438 \\
\text { two age group were } \\
\text { analyzed: } \square 80 \text { and } \geq 80 \\
\text { years old }\end{array}$ \\
\hline $\begin{array}{l}\text { Antoniou } \\
\text { et al. } \\
\text { Canada, 2011(34) }\end{array}$ & $\begin{array}{l}\text { Residents of } \\
\text { Ontario }\end{array}$ & $\begin{array}{l}\text { Population-based, } \\
\text { nested case- } \\
\text { control }\end{array}$ & $\begin{array}{l}\text { Study the risk of phenytoin toxicity } \\
\text { following the prescription of } \\
\text { trimethoprim/sulfamethoxazole among } \\
\text { older adults receiving phenytoin. }\end{array}$ & $\begin{array}{l}\mathrm{n}=3,944 \\
(796 \text { cases and } 3,148 \\
\text { controls) } \\
\text { Cases: } 75 \text { years (IQR:70- } \\
\text { 80) } \\
\text { Controls: } 74 \text { years } \\
\text { (IQR: } 70-80 \text { ) }\end{array}$ \\
\hline $\begin{array}{l}\text { Budnitz } \\
\text { et al. } \\
\text { U.S, 2011(35) }\end{array}$ & $\begin{array}{l}\text { National Electronic } \\
\text { Injury Surveillance } \\
\text { System-- } \\
\text { Cooperative } \\
\text { Adverse Drug } \\
\text { Event Surveillance } \\
\text { project }\end{array}$ & Surveillance case & $\begin{array}{l}\text { Estimate the frequency and rates } \\
\text { of hospitalization after emergency } \\
\text { department visits for ADR in older } \\
\text { adults and to assess the contribution } \\
\text { of specific medications, including } \\
\text { those identified as high-risk or } \\
\text { potentially inappropriate by national } \\
\text { quality measures. }\end{array}$ & $\begin{array}{l}\mathrm{n}=5,077 \\
\geq 65 \text { years } \\
\text { of age or older }\end{array}$ \\
\hline $\begin{array}{l}\text { Coupland } \\
\text { et al. } \\
\text { UK, 2011(36) }\end{array}$ & $\begin{array}{l}570 \text { general } \\
\text { practices in the } \\
\text { United Kingdom } \\
\text { supplying data to } \\
\text { the QResearch } \\
\text { primary care } \\
\text { database }\end{array}$ & Cohort study & $\begin{array}{l}\text { Investigate the association between } \\
\text { antidepressant treatment and risk of } \\
\text { several potential adverse outcomes } \\
\text { in older people with depression } \\
\text { and to examine risks by class of } \\
\text { antidepressant, duration of use, and } \\
\text { dose. }\end{array}$ & $\begin{array}{l}n=60,746 \\
75 \text { years } \\
\text { (SD: } 7.6 \text { ) }\end{array}$ \\
\hline $\begin{array}{l}\text { Chan et al. } \\
\text { Taiwan, } 2012^{(37)}\end{array}$ & $\begin{array}{l}\text { Medication Safety } \\
\text { Review Clinic } \\
\text { patients }\end{array}$ & Intervention study & $\begin{array}{l}\text { Report baseline drug-related problem } \\
\text { found in outpatient clinic participants, } \\
\text { as well as the correlates of having at } \\
\text { least one drug-related problem in this } \\
\text { population. }\end{array}$ & $\begin{array}{l}\mathrm{n}=193 \\
76.2 \text { years } \\
(\mathrm{SD}: 6.2)\end{array}$ \\
\hline
\end{tabular}




\begin{tabular}{|c|c|c|c|c|}
\hline Source & Setting & Design & Aim & $\begin{array}{c}\text { Sample } \\
\left.\text { Median age (IQR* or } \mathrm{SD}^{\dagger}\right)\end{array}$ \\
\hline $\begin{array}{l}\text { Baillargeon } \\
\text { et al. } \\
\text { U.S, } 2012^{(38)}\end{array}$ & $\begin{array}{l}\text { National sample } \\
\text { of Medicare } \\
\text { beneficiaries with } \\
\text { Part D benefits }\end{array}$ & $\begin{array}{l}\text { Case-control study } \\
\text { nested }\end{array}$ & $\begin{array}{l}\text { Examined the risk of bleeding } \\
\text { associated with exposure to all } \\
\text { antibiotic agents combined as well } \\
\text { as several specific antibiotic agents } \\
\text { in a cohort of older patients receiving } \\
\text { warfarin. }\end{array}$ & $\begin{array}{l}n=38,762 \\
\geq 65 \text { years }\end{array}$ \\
\hline $\begin{array}{l}\text { O'Connor } \\
\text { et al. } \\
\text { Ireland, 2012 }\end{array}$ & $\begin{array}{l}\text { An } 810 \text { bed } \\
\text { university teaching } \\
\text { hospital }\end{array}$ & Prospective study & $\begin{array}{l}\text { Examine the GerontoNet ADR } \\
\text { risk score in terms of its clinical } \\
\text { applicability, and its ability to } \\
\text { predict ADRs in hospitalized older } \\
\text { patients when compared with expert } \\
\text { judgments; and, identify other } \\
\text { variables that would influence the } \\
\text { presence of ADRs. }\end{array}$ & $\begin{array}{l}\mathrm{n}=513 \\
77 \text { years } \\
\text { (IQR: } 72-82)\end{array}$ \\
\hline $\begin{array}{l}\text { Somers et al. } \\
\text { Belgium, } 2012^{(40)}\end{array}$ & $\begin{array}{l}\text { Geriatric ward } \\
\text { of the Ghent } \\
\text { University } \\
\text { Hospital }\end{array}$ & $\begin{array}{l}\text { Retrospective, } \\
\text { observational study }\end{array}$ & $\begin{array}{l}\text { Evaluate the applicability of an } \\
\text { adapted version of the Medication } \\
\text { Appropriateness Index in geriatric } \\
\text { inpatients at the time of admission. }\end{array}$ & $\begin{array}{l}\mathrm{n}=50 \\
83.0 \text { years } \\
\text { (SD: } 6.2)\end{array}$ \\
\hline $\begin{array}{l}\text { Bakken et al. } \\
\text { Norway, } 2012^{(41)}\end{array}$ & $\begin{array}{l}\text { Intermediate-care } \\
\text { nursing home } \\
\text { unit (INHU) and } \\
\text { hospital wards } \\
\text { (HW) }\end{array}$ & $\begin{array}{l}\text { Observational } \\
\text { study }\end{array}$ & $\begin{array}{l}\text { Identify inappropriate prescribing } \\
\text { among older patients on admission to } \\
\text { and discharge from an INHU and HW, } \\
\text { and to compare changes during stay } \\
\text { within and between these groups. }\end{array}$ & $\begin{array}{l}\mathrm{n}=290 \\
84.7 \text { years } \\
\text { (SD: } 6.2 \text { ) }\end{array}$ \\
\hline $\begin{array}{l}\text { Romana } \\
\text { et al. } \\
\text { India, 2012 }\end{array}$ & $\begin{array}{l}\text { Victoria hospital } \\
\text { attached to } \\
\text { Bangalore Medical } \\
\text { College and } \\
\text { Research Institute }\end{array}$ & $\begin{array}{l}\text { Prospective } \\
\text { observational } \\
\text { hospital } \\
\text { based study }\end{array}$ & $\begin{array}{l}\text { Identify the ADRs occurring in the } \\
\text { older adults because of polypharmacy } \\
\text { and also assess the rationality of } \\
\text { prescription based on World Health } \\
\text { Organization criteria and Beers' } \\
\text { criteria. }\end{array}$ & $\begin{array}{l}\mathrm{n}=100 \\
66 \% \text { patients } \\
(60-69 \text { years), } 24 \%(70-79 \\
\text { years), } 10 \%(80-89 \text { years })\end{array}$ \\
\hline $\begin{array}{l}\text { Halvorsen } \\
\text { et al. } \\
\text { Norway, 2012(43) }\end{array}$ & $\begin{array}{l}\text { Nursing homes } \\
\text { (NHs) and home } \\
\text { nursing services } \\
\text { (HNS) }\end{array}$ & $\begin{array}{l}\text { Cross-sectional } \\
\text { study }\end{array}$ & $\begin{array}{l}\text { Examine and compare the quality of } \\
\text { drug prescribing for older patients in } \\
\mathrm{NHs} \text { and HNS. }\end{array}$ & $\begin{array}{l}n=11,254 \\
(N H s n=2,986) \\
(H N S n=8,268)\end{array}$ \\
\hline $\begin{array}{l}\text { Mehuys } \\
\text { et al. } \\
\text { Belgium, 2012(44) }\end{array}$ & $\begin{array}{l}\text { Community } \\
\text { pharmacies }(n=86)\end{array}$ & $\begin{array}{l}\text { Cross-sectional } \\
\text { observational study }\end{array}$ & $\begin{array}{l}\text { Examine: (a) types of drugs used } \\
\text { and presence of DDIs, (b) drug } \\
\text { adherence, (c) patient knowledge of } \\
\text { the purpose of their drugs and (d) } \\
\text { practical drug management capacity. }\end{array}$ & $\begin{array}{l}\mathrm{n}=338 \\
\text { home-dwelling older } \\
\text { adults using at least one } \\
\text { chronic medicine }\end{array}$ \\
\hline $\begin{array}{l}\text { Pincus et al. } \\
\text { Canada, } \\
2012^{(45)}\end{array}$ & $\begin{array}{l}\text { Residents of } \\
\text { Ontario }\end{array}$ & $\begin{array}{l}\text { Population-based } \\
\text { nested case- } \\
\text { control }\end{array}$ & $\begin{array}{l}\text { Examine the clinical consequences } \\
\text { of the possible DDI between } \\
\text { levothyroxine and warfarin. }\end{array}$ & $\begin{array}{l}\mathrm{n}=51,225 \\
80 \text { years } \\
\text { (IQR: } 75-86)\end{array}$ \\
\hline $\begin{array}{l}\text { Marcum } \\
\text { et al. } \\
\text { U.S, } 2012^{(46)}\end{array}$ & $\begin{array}{l}\text { Veterans Affairs } \\
\text { Medical Centers }\end{array}$ & $\begin{array}{l}\text { Retrospective } \\
\text { cohort }\end{array}$ & $\begin{array}{l}\text { Describe the prevalence of unplanned } \\
\text { hospitalizations caused by ADRs in } \\
\text { older veterans, and to examine the } \\
\text { association between this outcome } \\
\text { and polypharmacy after controlling } \\
\text { for comorbidities and other patient } \\
\text { characteristics. }\end{array}$ & $\begin{array}{l}n=678 \\
76.4 \text { years }\end{array}$ \\
\hline $\begin{array}{l}\text { Vila et al. } \\
\text { Spain, 2012 }\end{array}$ & $\begin{array}{l}\text { Eleven pharmacies } \\
\text { in the community of } \\
\text { Valencia }\end{array}$ & $\begin{array}{l}\text { Single blind } \\
\text { controlled study }\end{array}$ & $\begin{array}{l}\text { Improve the use of benzodiazepines } \\
\text { in the older adults by pharmacist- } \\
\text { physician collaboration, and to insure } \\
\text { that are effective, safe, and used as } \\
\text { long as necessary. }\end{array}$ & $\begin{array}{l}n=314 \text { patients } \\
65-74 \text { years }\end{array}$ \\
\hline $\begin{array}{l}\text { Obreli-Neto et al. } \\
\text { Brazil, } 2012^{(48)}\end{array}$ & $\begin{array}{l}\text { Primary public } \\
\text { healthcare system } \\
\text { in the Ourinhos } \\
\text { micro-region in } \\
\text { Brazil }\end{array}$ & $\begin{array}{l}\text { Prospective cohort } \\
\text { study }\end{array}$ & $\begin{array}{l}\text { Investigate the incidence of DDIs } \\
\text { related to ADRs in older outpatients } \\
\text { who attended public primary } \\
\text { healthcare units in a southeastern } \\
\text { region of Brazil; and, to investigate } \\
\text { the possible predictors of DDI-related } \\
\text { ADRs. }\end{array}$ & $\begin{array}{l}\mathrm{n}=443 \text { patients } \\
\text { Exposed ( } \mathrm{n}=28,80.3 \pm 7.1 \\
\text { years) and non-exposed } \\
\text { group ( } \mathrm{n}=405,65.9 \pm 3.4 \\
\text { years) }\end{array}$ \\
\hline
\end{tabular}




\begin{tabular}{|c|c|c|c|c|}
\hline Source & Setting & Design & Aim & $\begin{array}{c}\text { Sample } \\
\left.\text { Median age (IQR* or } \text { SD }^{\dagger}\right)\end{array}$ \\
\hline $\begin{array}{l}\text { Obreli-Neto et al. } \\
\text { Brazil, 2012 }\end{array}$ & $\begin{array}{l}\text { Five Brazilian } \\
\text { cities located in the } \\
\text { Ourinhos Micro- } \\
\text { region, Sao Paulo } \\
\text { State }\end{array}$ & $\begin{array}{l}\text { Cross-sectional } \\
\text { study }\end{array}$ & $\begin{array}{l}\text { Investigate the prevalence of clinically } \\
\text { important potential DDIs in older } \\
\text { patients attending the public primary } \\
\text { health care system in Brazil; and, } \\
\text { to investigate possible predictors of } \\
\text { potential DDIs. }\end{array}$ & $\begin{array}{l}n=12,343 \text { Exposed } \\
(n=5,85563.31 \pm 3.47 \\
\text { years) and unexposed } \\
\text { group }(n=6,488,61.42 \\
\pm 2.25 \text { years })\end{array}$ \\
\hline $\begin{array}{l}\text { Ramanath and } \\
\text { Nedmballi } \\
\text { India, 2012(50) }\end{array}$ & $\begin{array}{l}\text { Medicine } \\
\text { Department of } \\
\text { Adichunchanagiri } \\
\text { Hospital and } \\
\text { Research Centre, } \\
\text { B. G. Nagara }\end{array}$ & $\begin{array}{l}\text { Prospective, } \\
\text { observational study }\end{array}$ & Uniformed. & $\begin{array}{l}\mathrm{n}=163 \\
77.9 \% 60-70 \text { years } \\
19.0 \% 71-80 \text { years } \\
3.1 \% 81-90 \text { years }\end{array}$ \\
\hline $\begin{array}{l}\text { Bonnet-Zamponi } \\
\text { et al. } \\
\text { France, } 2013^{(51)}\end{array}$ & $\begin{array}{l}\text { Six acute geriatric } \\
\text { units in Paris and } \\
\text { suburbs }\end{array}$ & $\begin{array}{l}\text { Prospective, } \\
\text { randomized, } \\
\text { parallel-group, } \\
\text { open-label trial }\end{array}$ & $\begin{array}{l}\text { Assess the effect of an intervention } \\
\text { on drug-related problem (ADR, } \\
\text { adherence problems, underuse), } \\
\text { readmission rates in older adults. }\end{array}$ & $\begin{array}{l}\mathrm{n}=665 \text { (intervention group } \\
\mathrm{n}=317 \text { and control group } \\
\mathrm{n}=348 \text { ) } \\
86.1 \pm 6.2 \text { years }\end{array}$ \\
\hline $\begin{array}{l}\text { Nickel et al. } \\
\text { Switzerland, } \\
2013^{(52)}\end{array}$ & $\begin{array}{l}\text { Emergency } \\
\text { department of the } \\
\text { University } \\
\text { Hospital Basel }\end{array}$ & $\begin{array}{l}\text { Cross-sectional } \\
\text { diagnostic study }\end{array}$ & $\begin{array}{l}\text { Identify the frequency of drug-related } \\
\text { problem among patients presenting } \\
\text { to the emergency department with } \\
\text { non-specific complaints, such as } \\
\text { generalized weakness and to evaluate } \\
\text { responsible drug classes. }\end{array}$ & $\begin{array}{l}\mathrm{n}=633 \\
81 \text { years } \\
\text { (IQR: 72-87) }\end{array}$ \\
\hline $\begin{array}{l}\text { Marusic } \\
\text { et al. } \\
\text { Croatia, 2013(53) }\end{array}$ & $\begin{array}{l}\text { Internal Medicine } \\
\text { Clinic of University } \\
\text { Hospital } \\
\text { Dubrava, Zagreb }\end{array}$ & $\begin{array}{l}\text { Prospective } \\
\text { observational study }\end{array}$ & $\begin{array}{l}\text { Evaluate the incidence and type of } \\
\text { actual DDIs that result in ADRs or } \\
\text { diminished therapeutic effect in older } \\
\text { adults within } 30 \text { days of discharge } \\
\text { from an internal medicine clinic. }\end{array}$ & $\begin{array}{l}n=222 \\
72 \text { (IQR: 65-91) }\end{array}$ \\
\hline $\begin{array}{l}\text { Bondesson } \\
\text { et al. } \\
\text { Sweden, 2013(54) }\end{array}$ & $\begin{array}{l}\text { Internal medicine } \\
\text { ward at the } \\
\text { University Hospital } \\
\text { of Lund }\end{array}$ & $\begin{array}{l}\text { Prospective two- } \\
\text { period study }\end{array}$ & $\begin{array}{l}\text { Examine the impact of a new model } \\
\text { of care, in which a clinical pharmacist } \\
\text { conducts structured medication } \\
\text { reviews and a multi-professional team } \\
\text { collates systematic medication care } \\
\text { plans, on the number of unidentified } \\
\text { drug-related problem. }\end{array}$ & $\begin{array}{l}\mathrm{n}=141 \\
\text { (intervention group } \\
\mathrm{n}=70,81,9 \pm 7.5 \text { years } \\
\text { and control group } \mathrm{n}=71 \text {, } \\
81.3 \pm 6.5 \text { years) }\end{array}$ \\
\hline $\begin{array}{l}\text { Yeoh et al. } \\
\text { Singapore, } \\
2013^{(55)}\end{array}$ & $\begin{array}{l}\text { National Cancer } \\
\text { Centre Singapore }\end{array}$ & Prospective study & $\begin{array}{l}\text { Identify common drug-related problem } \\
\text { among older cancer patients, to } \\
\text { determine the effectiveness of } \\
\text { medication therapy management } \\
\text { service in resolving drug-related } \\
\text { problem, to determine the clinical } \\
\text { significance of pharmacist } \\
\text { interventions, and to determine } \\
\text { patients' satisfaction level. }\end{array}$ & $\begin{array}{l}n=118 \\
71.7 \text { years } \\
\text { (range 65-86). }\end{array}$ \\
\hline $\begin{array}{l}\text { Klopotowska } \\
\text { et al. } \\
\text { Netherland, } \\
2013^{(56)}\end{array}$ & $\begin{array}{l}\text { Internal Medicine } \\
\text { wards of three } \\
\text { hospitals }\end{array}$ & Cohort study & $\begin{array}{l}\text { Assess medical teams' ability to } \\
\text { recognize adverse drug events in } \\
\text { older inpatients. }\end{array}$ & $\begin{array}{l}n=250 \\
76.9 \pm 7.5 \text { years }\end{array}$ \\
\hline $\begin{array}{l}\text { Somers } \\
\text { et al. } \\
\text { Belgium, 2013(57) }\end{array}$ & $\begin{array}{l}\text { Geriatric ward } \\
\text { of the Ghent } \\
\text { University Hospital }\end{array}$ & $\begin{array}{l}\text { Observational } \\
\text { monocentric study }\end{array}$ & $\begin{array}{l}\text { Evaluate the type, acceptance rate, } \\
\text { and clinical relevance of clinical } \\
\text { pharmacist recommendations. }\end{array}$ & $\begin{array}{l}\mathrm{n}=100 \\
81.4 \text { years } \\
\text { (SD: } 6.65 ; \text { median: } 82 \\
\text { years) }\end{array}$ \\
\hline
\end{tabular}

Figure 2 - Articles revised according to their characteristics. Brasilia, Federal District, Brazil, 2014 *IQR - Interquatile Range; TSD - Standard Derivation

\begin{tabular}{|c|c|}
\hline Source & \multicolumn{1}{c|}{ Results of studies } \\
\hline $\begin{array}{c}\text { Gallagher and } \\
\text { O'Mohony } \\
\text { Ireland, 2008(1) }\end{array}$ & $\begin{array}{l}\text { Tool of Older Persons' potentially inappropriate Prescriptions criteria identified } 336 \text { potentially inappropriate medicines } \\
\text { affecting 247 patients }(35 \%), \text { of whom one-third }(n=82) \text { presented with an associated ADR. Beers' criteria identified 226 } \\
\text { potentially inappropriate medicines affecting 177 patients (25\%), of whom 43 presented an associated ADR. }\end{array}$ \\
\hline
\end{tabular}




\begin{tabular}{|c|c|}
\hline Source & Results of studies \\
\hline $\begin{array}{l}\text { Gallagher } \\
\text { et al. } \\
\text { Ireland, } 2008^{(12)}\end{array}$ & $\begin{array}{l}\text { Patients taking }>5 \text { medications were } 3.3 \text { times more likely to receive an inappropriate medication than those taking } \\
\leq 5 \text { medications. } 49 \% \text { of patients with inappropriate prescriptions were admitted to hospital with adverse effects to the } \\
\text { inappropriate medications. There was no association between gender and age. }\end{array}$ \\
\hline $\begin{array}{l}\text { Zhang et al. } \\
\text { Australia, 2009(13) }\end{array}$ & $\begin{array}{l}5,056(17.7 \%) \text { patients had a repeat admission for an ADR. Comorbid congestive cardiac failure, peripheral vascular } \\
\text { disease, chronic pulmonary disease, rheumatologic disease, mild liver disease, moderate to severe liver disease, } \\
\text { moderate diabetes, diabetes with chronic complications, renal disease, any malignancy including lymphoma and } \\
\text { leukemia, and metastatic solid tumors were strong predictive factors. }\end{array}$ \\
\hline $\begin{array}{l}\text { Juurlink et al. } \\
\text { Canada, 2009(14) }\end{array}$ & $\begin{array}{l}\text { Among } 13,636 \text { patients receiving clopidogrel following acute myocardial infarction, } 734 \text { cases were identified, readmitted } \\
\text { with myocardial infarction and 2,057 controls. The use of proton pump inhibitors was associated with an increased risk } \\
\text { of re-infarction. }\end{array}$ \\
\hline $\begin{array}{l}\text { Rassen et al. } \\
\text { U.S, } 2009^{(15)}\end{array}$ & $\begin{array}{l}\text { On a pooled basis, } 2.6 \% \text { of those who also initiated a proton pump inhibitors vs } 2.1 \% \text { of proton pump inhibitors non } \\
\text { users had a myocardial infarction hospitalization; } 1.5 \% \text { vs } 0.9 \% \text { died, and } 3.4 \% \text { vs } 3.1 \% \text { underwent revascularization. }\end{array}$ \\
\hline $\begin{array}{l}\text { Miquel et al. } \\
\text { Spain, 2010(16) }\end{array}$ & $\begin{array}{l}\text { Screening Tool of Older Persons' potentially inappropriate Prescriptions criteria detected potentially inappropriate } \\
\text { medicines in } 36 \% \text { (primary care), } 54 \% \text { (hospital geriatric assessment outpatient clinic) and } 50 \% \text { (nursing home) of } \\
\text { the subjects. Beers' criteria detected potentially inappropriate drugs in } 24 \% \text { (primary care), } 26 \% \text { (hospital geriatric } \\
\text { assessment outpatient clinic) and } 20 \% \text { (nursing home) of the subjects. }\end{array}$ \\
\hline $\begin{array}{l}\text { Weir et al. } \\
\text { Canada, 2010 }\end{array}$ & $\begin{array}{l}\text { Compared with amoxicillin, the use of trimethoprim/sulfamethoxazole was associated with a substantially greater risk of } \\
\text { hyperkalemia requiring hospital admission. When dosing was considered, the association was greater at higher doses } \\
\text { of trimethoprim/sulfamethoxazole. When the primary analysis was repeated in a cohort of non } \beta \text {-blocker users, the risk } \\
\text { of hyperkalemia comparing trimethoprim/sulfamethoxazole to amoxicillin was not significantly different from that found } \\
\text { among } \beta \text {-blocker users. }\end{array}$ \\
\hline $\begin{array}{l}\text { Kelly et al. } \\
\text { Canada, 2010 }\end{array}$ & $\begin{array}{l}\text { Of } 2,430 \text { women treated with tamoxifen and a single selective serotonin reuptake inhibitor, } 374(15.4 \%) \text { died of breast } \\
\text { cancer during follow-up. After adjustment for age, duration of tamoxifen treatment, and other potential confounders, } \\
\text { absolute increases of } 25 \%, 50 \% \text {, and } 75 \% \text { in the proportion of time on tamoxifen with overlapping use of paroxetine } \\
\text { were associated with } 24 \%, 54 \% \text {, and } 91 \% \text { increases in the risk of death from breast cancer. }\end{array}$ \\
\hline $\begin{array}{l}\text { Somers et al. } \\
\text { Australia, } 2010^{(19)}\end{array}$ & $\begin{array}{l}\text { Polypharmacy was identified in } 91.2 \% .50 .4 \% \text { were found to be taking at least one potentially inappropriate medication. } \\
\text { The combination of antipsychotics and antidepressants was the most frequently observed DDI. }\end{array}$ \\
\hline $\begin{array}{l}\text { Harugeri } \\
\quad \text { et al. } \\
\text { India, } 2010^{(20)}\end{array}$ & $\begin{array}{l}\text { Polypharmacy was observed in } 44.5 \% \text { and } 90.4 \% \text { of patients at admission and during hospital stay. A total of } 360 \text { ADRs } \\
\text { were observed in } 292 \text { patients. The increased number }(\geq 9) \text { of concurrent medications' use during hospital stay was } \\
\text { found as the only influential predictor of potentially inappropriate medication use. Gender, age, number of diseases and } \\
\text { number of concurrent medications used at admissions were not predictors' factors. }\end{array}$ \\
\hline $\begin{array}{l}\text { Bacic-Vrca } \\
\quad \text { et al. } \\
\text { Croatia, } 2010^{(21)}\end{array}$ & $\begin{array}{l}\text { Were identified } 215 \text { drug combinations with the potential to cause clinically significant interaction, out of which } 83.3 \% \\
\text { had clinical significance C (specified agents may interact in a clinically significant manner; monitoring therapy is } \\
\text { suggested), } 16.3 \% \text { clinical significance D (the two medications may interact in a clinically significant manner), and } 0.4 \% \\
\text { clinical significance X (contraindicated combination). }\end{array}$ \\
\hline $\begin{array}{l}\text { Rahmawati } \\
\quad \text { et al. } \\
\text { Indonesia, } 2010^{(22)}\end{array}$ & $\begin{array}{l}\text { Of the } 100 \text { cases, } 65 \% \text { cases had experienced potential DDIs range from } 1 \text { to } 17 \text {. Of total } 204 \text { DDIs incidences, } 25 \% \\
\text { were of significance level } 1 \text { and } 39 \% \text { of significance level } 2 \text {. The study showed that the number of potential DDIs } \\
\text { increased as the number of medications used per day increased. Geriatric patients taking nine or more medication } \\
\text { tended to have more DDIs }(6.8 \pm 5.5) \text { in comparison to those with one to two medications. }\end{array}$ \\
\hline $\begin{array}{l}\text { Wright et al. } \\
\text { Canada, 2011 }\end{array}$ & $\begin{array}{l}\text { Of the } 7,100 \text { patients admitted to hospital because of hypotension while receiving a calcium-channel blocker, } 176 \\
\text { had been prescribed a macrolide antibiotic during either the risk or control intervals. Erythromycin was most strongly } \\
\text { associated with hypotension, followed by clarithromycin. }\end{array}$ \\
\hline $\begin{array}{l}\text { Steinman } \\
\text { et al. } \\
\text { U.S, } 2011^{(24)}\end{array}$ & $\begin{array}{l}\text { Over the one-year study period, } 126 \text { patients }(33 \%) \text { developed } 167 \text { adverse drug events. The risk of adverse drug } \\
\text { events was not associated with any of the geriatric conditions, and the strongest factor associated with adverse drug } \\
\text { events was the number of drugs added to a patient's medication regimen during the } 1 \text {-year's study period. }\end{array}$ \\
\hline $\begin{array}{l}\text { Steinman } \\
\text { et al. } \\
\text { US, } 2011^{(25)}\end{array}$ & $\begin{array}{l}\text { Over the 12-month follow-up period, } 497 \text { ADRs occurred in } 269 \text { participants, including } 187 \text { ADRs considered preventable } \\
\text { and } 127 \text { considered severe. Many geriatric conditions were not associated with risk of ADRs. }\end{array}$ \\
\hline $\begin{array}{l}\text { Santos et al. } \\
\text { Brazil, 2011(26) }\end{array}$ & $\begin{array}{l}\text { The } 36 \text { possible ADRs found were related to gastrointestinal tract, skin and the nervous system. A total of } 63 \text { DDIs were } \\
\text { found on patient prescriptions. }\end{array}$ \\
\hline $\begin{array}{l}\text { Mendes-Netto } \\
\quad \text { et al. } \\
\text { Brazil, } 2011^{(27)}\end{array}$ & Prescriptions with 2 to $3(39 \%), 4$ to $5(88.8 \%)$ and 6 to $7(100 \%)$ medications showed potential DDIs. \\
\hline
\end{tabular}




\begin{tabular}{|c|c|}
\hline Source & Results of studies \\
\hline $\begin{array}{c}\text { Antoniou } \\
\text { et al. } \\
\text { Canada, 2011(28) }\end{array}$ & $\begin{array}{l}\text { During the } 18 \text { year study period, } 6,903 \text { admissions for hyperkalemia were identified, } 306 \text { of which occurred within } 14 \\
\text { days of antibiotic use. Of these, } 248(81 \%) \text { cases were matched to } 783 \text { controls. } 10.8 \% \text { of spironolactone users received } \\
\text { at least one prescription for trimethoprim/sulfamethoxazole. Compared with amoxicillin, prescription of trimethoprim/ } \\
\text { sulfamethoxazole was associated with a marked increase in the risk of admission to hospital for hyperkalemia. }\end{array}$ \\
\hline $\begin{array}{l}\text { Varallo et al. } \\
\text { Brazil, 2011(29) }\end{array}$ & $\begin{array}{l}\text { Were reported } 167 \text { different drugs, of which } 58 \text { were responsible for } 99 \text { ADRs. Of these ADRs, } 4 \text { were related to the use } \\
\text { of potentially inappropriate medicines, } 82 \text { to the use of drugs other than potentially inappropriate medicines and } 13 \text { of } \\
\text { both categories of drugs (potentially inappropriate medicines and non potentially inappropriate medicines). The majority } \\
(57.6 \%) \text { of ADRs identified were classified as "possible". }\end{array}$ \\
\hline $\begin{array}{l}\text { Gallagher et al. } \\
\text { Ireland, } 2011^{(30)}\end{array}$ & $\begin{array}{l}\text { Prescription of medications with potential for clinically important DDIs was significantly reduced in the intervention group } \\
\text { at discharge and during follow-up. }\end{array}$ \\
\hline $\begin{array}{l}\text { Hanlon et al. } \\
\text { U.S, 2011(11) }\end{array}$ & $\begin{array}{l}\text { Among depressed patients who received antidepressants, } 43.1 \% \text { had potential inappropriate use due primarily to } \\
\text { problems seen with drug-drug and drug-disease interactions. }\end{array}$ \\
\hline $\begin{array}{l}\text { Skaar and O'Connor } \\
\quad \text { U.S, 2011(32) }\end{array}$ & $\begin{array}{l}\text { Were identified } 25 \text { DDIs of clinical importance in ambulatory settings. The potential serious interactions included aged } \\
\geq 85 \text { years, annual income }>\$ 50,000 \text { and higher numbers of prescriptions: } 8-10 \text { and } \geq 11 \text { drugs. }\end{array}$ \\
\hline $\begin{array}{l}\text { Venturini } \\
\text { et al. } \\
\text { Brazil, 2011(33) }\end{array}$ & $\begin{array}{l}\text { Number of interactions: Average: } 1.19 \text { (SD: } 2.23) \\
\text { Intensity of interaction: Low: } 17 \text { (8.8\%); Moderate: } 135 \text { (69.9\%); Severe: } 41 \text { (21.2\%). }\end{array}$ \\
\hline $\begin{array}{c}\text { Antoniou } \\
\text { et al. } \\
\text { Canada, } 2011^{(34)}\end{array}$ & $\begin{array}{l}\text { Among } 58,429 \text { older people receiving phenytoin, were identified } 796 \text { case patients hospitalized for phenytoin toxicity } \\
\text { and } 3,148 \text { matched controls. Following multivariable adjustment for potential confounders, were observed a more } \\
\text { than doubling of the risk of phenytoin toxicity following the receipt of trimethoprim/sulfamethoxazole. In contrast, were } \\
\text { observed no such risk with amoxicillin. }\end{array}$ \\
\hline $\begin{array}{l}\text { Budnitz et al. } \\
\text { U.S, 2011(35) }\end{array}$ & $\begin{array}{l}\text { On the basis of } 5,077 \text { cases identified in the sample, there were an estimated } 99,628 \text { emergency hospitalizations for } \\
\text { adverse drug events in United States. Emergency department visits that resulted in hospitalization, as compared with } \\
\text { visits that did not result in hospitalization, were more likely to involve unintentional overdoses }(65.7 \% \text { vs. } 45.7 \%) \text { and } \\
\text { five or more concomitant medications }(54.8 \% \text { vs. } 39.9 \%) \text {. Four medications or medication classes were implicated } \\
\text { alone or in combination in } 67.0 \% \text { of hospitalizations: warfarin ( } 33.3 \%) \text {, insulins (13.9\%), oral antiplatelet agents }(13.3 \%) \text {, } \\
\text { and oral hypoglycemic agents }(10.7 \%) \text {. High-risk medications were implicated in only } 1.2 \% \text { of hospitalizations. }\end{array}$ \\
\hline $\begin{array}{l}\text { Coupland } \\
\text { et al. } \\
\text { U.K, 2011(36) }\end{array}$ & $\begin{array}{l}54,038(89.0 \%) \text { patients received at least one prescription for an antidepressant during follow-up. Selective serotonin } \\
\text { reuptake inhibitors were associated with the highest adjusted hazard ratios for falls and hyponatraemia compared } \\
\text { with when antidepressants were not being used. The group of other antidepressants was associated with the highest } \\
\text { adjusted hazard ratios for all cause mortality, attempted suicide/self harm, stroke/transient ischemic attack, fracture, and } \\
\text { epilepsy/seizures, compared with when antidepressants were not being used. }\end{array}$ \\
\hline
\end{tabular}

\begin{tabular}{|c|l|}
\hline $\begin{array}{c}\text { Chan et al. } \\
\text { Taiwan, } 2012^{(37)}\end{array}$ & $\begin{array}{l}\text { Problem domain: interactions (2 categories) (12\%). Category: potential interaction (12\%). Problem domain: adverse } \\
\text { reactions (9\%).Category: side effect suffered (non allergic) (9\%). }\end{array}$ \\
\hline
\end{tabular}

\begin{tabular}{|c|c|}
\hline $\begin{array}{c}\text { Baillargeon } \\
\text { et al. }\end{array}$ & $\begin{array}{c}\text { Exposure to any antibiotic agent within the } 15 \text { days of the event/index date was associated with an increased risk of } \\
\text { bleeding. All six specific antibiotic drug classes examined [azole antifungals, macrolides, quinolones, cotrimoxazole, } \\
\text { penicillins and cephalosporins] were associated with an increased risk of bleeding. }\end{array}$ \\
\hline $\begin{array}{c}\text { O'Connor } \\
\text { et al. }\end{array}$ & $\begin{array}{l}\text { In-hospital ADRs were identified in } 135 \text { patients (26\%). Variables which increased ADR risk include (i) renal failure, (ii) } \\
\text { increasing number of medications, (iii) inappropriate medications and (iv) age } \geq 75 \text { years. }\end{array}$
\end{tabular}
reland, $2012^{(39)}$

Somers et al. Belgium, 2012(40)
The number of drugs was significantly correlated with higher Medication Appropriateness Index scores. Good agreement between the scores of the geriatrician and the clinical pharmacist was found: the $\mathrm{k}$ values of the 8 questions ranged between 0.54 and 0.77 and the overall mean $\mathrm{K}$ value was 0.71 . Were found the highest $\mathrm{K}$ values for drug-disease interactions (0.77), dosage (0.74), and ADR (0.74), and the lowest values for directions $(0.54)$ and indication (0.65).
Bakken et al.

Norway, 2012(41)
The prevalence of potentially inappropriate medicines increased from $24 \%$ to $35 \%$; concomitant use of $\geq 3$ psychotropic/ opioid drugs and drug combinations including non-steroid anti-inflammatory drugs increased significantly. Serious DDI were scarce both on admission and discharge $(0.7 \%)$.
Romana et al. India, 2012(42)
The drugs involved in majority of the DDI included ciprofloxacin, theophylline, ceftriaxone and azithromycin. ADR were mainly seen in $15 \%$ of patients. Only certain and probable ADR were taken for analysis. ADR were mainly seen for insulin (5 patients), followed by salbutamol (3 patients), amlodipine ( 2 patients) and digoxin ( 2 patients). 


\begin{tabular}{|c|c|}
\hline Source & Results of studies \\
\hline $\begin{array}{l}\text { Halvorsen } \\
\quad \text { et al. } \\
\text { Norway, 2012(43) }\end{array}$ & $\begin{array}{l}\text { Were observed } 8,615 \text { DDIs in } 55 \% \text { of patients, } 48 \% \text { in nursing homes and } 57 \% \text { in home nursing services. More women } \\
\text { in home nursing services compared with men were exposed to DDI; however, no significant difference between genders } \\
\text { was found in nursing homes ( } 48.2 \% \text { vs. } 46.7 \%) \text {. The number of DDIs per patient was significantly correlated with the } \\
\text { number of drugs used and weakly inversely correlated with patient's age in both nursing homes and in home nursing } \\
\text { services. }\end{array}$ \\
\hline $\begin{array}{l}\text { Mehuys et al. } \\
\text { Belgium, 2012(44) }\end{array}$ & $\begin{array}{l}\text { The sample used a median of } 5 \text { chronic drugs per patient, and half used psychotropic medication chronically, mainly } \\
\text { benzodiazepines. In } 100 \text { patients }(29.6 \%) \text { at least one DDI of potential clinical significance was observed. The overall } \\
\text { mean adherence per patient was very high }(98.1 \%) \text {, but } 39.6 \% \text { of individuals were un-adherent with at least one } \\
\text { medication. The participants reported several practical problems with drug taking: difficulties with vision ( } 32.0 \%) \text {, blister } \\
\text { opening }(12.1 \%) \text {, tablet swallowing }(14.8 \%) \text {, tablet splitting }(29.7 \%) \text { and distinction between different drug packages } \\
(23.4 \%) \text {. }\end{array}$ \\
\hline $\begin{array}{l}\text { Pincus et al. } \\
\text { Canada, 2012 } \\
\end{array}$ & $\begin{array}{l}\text { Were found no increase in the risk of hemorrhage among older patients receiving warfarin who initiated levothyroxine in } \\
\text { the previous } 30 \text { days. As expected, were found no significant association with more distant exposures (adjusted Odds } \\
\text { ratio } 0.76,95 \% \text { confidence interval } 0.26-2.25 \text { for patients initiating levothyroxine } 31-60 \text { days before the index date; } \\
\text { adjusted Odds ratio } 0.67,95 \% \text { confidence interval } 0.15-3.01 \text { for patients initiating levothyroxine } 61-90 \text { days before } \\
\text { bleeding). }\end{array}$ \\
\hline $\begin{array}{l}\text { Marcum } \\
\text { et al. } \\
\text { U.S, } 2012^{(46)}\end{array}$ & $\begin{array}{l}\text { Seventy ADRs involving } 113 \text { drugs were found in } 68(10 \%) \text { hospitalizations of older veterans, of which } 25(36.8 \%) \text { were } \\
\text { preventable. The most common ADRs that occurred were bradycardia, hypoglycemia, falls, and mental status changes. } \\
\text { The polypharmacy }(\geq 9 \text { and } 5-8) \text { was associated with greater risk of ADR-related hospitalization. }\end{array}$ \\
\hline $\begin{array}{l}\text { Vila et al. } \\
\text { Spain, 2012 }\end{array}$ & $\begin{array}{l}\text { In } 83 \text { patients (they used } 99 \text { benzodiazepines) were detected } 132 \text { interactions: } 33 \% \text { with omeprazole, } 19 \% \text { anxiolytics, } \\
14 \% \text { beta-blockers, and } 8 \% \text { with other hypnotics followed of antidepressants, ketoconazole, fentanyl and ciprofloxacin, } \\
\text { among others. } 278 \text { ADR were found: } 32 \% \text { memory loss, } 21 \% \text { drowsiness, } 12 \% \text { lack of coordination, } 8.3 \% \text { dizziness } \\
\text { and confusion, } 8,3 \% \text { disorientation and } 10 \% \text { others. }\end{array}$ \\
\hline $\begin{array}{l}\text { Obreli-Neto } \\
\quad \text { et al. } \\
\text { Brazil, 2012(48) }\end{array}$ & $\begin{array}{l}\text { DDI-related ADRs were verified in } 6.5 \% \text { of the sample. Twenty-one drugs were involved, and warfarin was the most } \\
\text { common of these, involved in } 11 \text { of the DDI-related ADRs. Gastrointestinal bleeding was the most frequent DDI-related } \\
\text { ADR hospital admission was necessary in } 36.7 \% \text {. In the non-exposed group, } 48 \text { drugs were involved in potential DDIs. } \\
\text { The age, the number of diagnosed diseases, and the number of drugs consumed were associated with an increased } \\
\text { risk of DDI-related ADRs. The older adults who took five or more drugs had a significantly higher risk of DDI-related } \\
\text { ADRs than those who took three to four drugs. No significant differences were observed with regard to sex. }\end{array}$ \\
\hline $\begin{array}{l}\text { Obreli-Neto } \\
\quad \text { et al. } \\
\text { Brazil, 2012(49) }\end{array}$ & $\begin{array}{l}\text { The prevalence of clinically important potential DDIs found was } 47.4 \% \text {. Female sex, diagnosis of } \geq 3 \text { diseases, and } \\
\text { diagnosis of hypertension were associated with potential DDIs. Drug therapy regimens involving } \geq 2 \text { prescribers, } \geq 3 \\
\text { drugs, } \geq 2 \text { Anatomical Therapeutic Chemical codes, } \geq 2 \text { drugs acting on cytochrome P450, and Anatomical Therapeutic } \\
\text { Chemical codes 'B' (blood and blood-forming organs) and 'C' (cardiovascular system) were associated with potential } \\
\text { DDIs. }\end{array}$ \\
\hline $\begin{array}{l}\text { Ramanath and } \\
\text { Nedmballi } \\
\text { India, 2012(50) }\end{array}$ & $\begin{array}{l}\text { DDI were reported only in } 8 \%(n=13) \text {. Inpatient } 11.4 \%(n=9) \text { and outpatient } 4.8 \%(n=4) \text {. No ADR were observed during } \\
\text { the study. }\end{array}$ \\
\hline $\begin{array}{l}\text { Bonnet-Zamponi } \\
\quad \text { et al. } \\
\text { France, } 2013^{(51)}\end{array}$ & $\begin{array}{l}\text { ADRs were directly responsible or contributed to } 27(28.7 \%) \text { and } 13(17.3 \%) \text { of definite readmissions in the control group } \\
\text { and intervention group, respectively. Antithrombotic, antihypertensive, and psychotropic drugs were implicated in } 81.5 \% \\
(22 / 27) \text { and } 61.5 \%(8 / 13) \text { of the ADR-related readmissions in the control group and intervention group, respectively. }\end{array}$ \\
\hline $\begin{array}{l}\text { Nickel et al. } \\
\text { Switzerland, } \\
2013^{(52)}\end{array}$ & $\begin{array}{l}\text { A total number of } 77 \text { patients with drug-related problems were identified, contributing to } 12.2 \% \text { of the total study } \\
\text { population ( } 633 \text { patients). Forty-two drug-related problems ( } 56 \% \text { of all drug-related problems) were classified under the } \\
\text { P1 domain "adverse drug reactions". }\end{array}$ \\
\hline $\begin{array}{l}\text { Marusic et al. } \\
\text { Croatia, 2013(53) }\end{array}$ & $\begin{array}{l}\text { Potential DDIs were identified in } 190 \text { ( } 85.6 \%) \text { patients. Actual DDIs were detected in } 21(9.5 \%) \text { patients. In } 19 \text { patients, } \\
\text { DDIs resulted in an ADR. Angiotensin-converting enzyme inhibitors were the drug class most frequently associated with } \\
\text { DDI-related ADRs. }\end{array}$ \\
\hline $\begin{array}{l}\text { Bondesson } \\
\quad \text { et al. } \\
\text { Sweden, } 2013^{(54)}\end{array}$ & $\begin{array}{l}\text { A total of } 690 \text { drug-related problem per patient were identified (mean=9.9), } 393(57 \%) \text { were classified as actual drug- } \\
\text { related problem per patient and } 297(43 \%) \text { were classified as potential drug-related problem per patient. The three } \\
\text { most common types identified were: wrong drug ( } n=151) \text {, unnecessary drug therapy }(n=136) \text { and adverse drug reaction } \\
(n=118) \text {. }\end{array}$ \\
\hline $\begin{array}{l}\text { Yeoh et al. } \\
\text { Singapore, } \\
2013^{(55)}\end{array}$ & $\begin{array}{l}361 \text { drug-related problems were detected. The most common types were: potential DDIs }(32.4 \%) \text {, ADR }(31.6 \%) \text {, and } \\
\text { patient non-adherence }(13.3 \%) \text {. Approximately half }(54.0 \%) \text { of the potential DDIs detected were pharmacokinetic } \\
\text { interactions. Majority }(85.1 \%) \text { of the ADR detected in this study was associated with chemotherapy. }\end{array}$ \\
\hline
\end{tabular}




\begin{tabular}{|c|c|}
\hline Source & Results of studies \\
\hline $\begin{array}{l}\text { Klopotowska } \\
\text { et al. } \\
\text { Netherlands, } \\
2013^{(56)}\end{array}$ & $\begin{array}{l}\text { The medical teams did not recognize } 19.9 \% \text { of all ADRs present upon admission and } 20.3 \% \text { of all ADRs occurring } \\
\text { during the hospital stay. Unrecognized ADRs were significantly more often ADRs with possible causality, ADRs caused } \\
\text { by medication errors and ADRs not manifesting as new symptoms. The medical teams did not recognize } 23.2 \% \text { of mild } \\
\text { to moderately severe ADRs and } 16.5 \% \text { of severe, life-threatening, or fatal ADRs. }\end{array}$ \\
\hline $\begin{array}{l}\text { Somers et al. } \\
\text { Belgium, 2013 }\end{array}$ & $\begin{array}{l}\text { The clinical pharmacist recommended } 304 \text { drug therapy changes for } 100 \text { patients taking a total of } 1,137 \text { drugs. The most } \\
\text { common underlying drug-related problem concerned incorrect dose ( } 31 \%) \text {, DDI ( } 20 \%) \text {, and ADR (15\%) which appeared } \\
\text { most frequently for cardiovascular drugs, drugs for the central nervous system, and drugs for the gastrointestinal tract. } \\
\text { The criteria with the highest (most inappropriate) scores were DDI, dosage, and drug choice, both for the calculation } \\
\text { before and after the recommendation. }\end{array}$ \\
\hline
\end{tabular}

Figure 3 - Summary of results of studies on potential DDI and ADR. Brasilia, Federal District, Brazil, 2014

Of the 47 records resulting from the search strategies, $24(51.1 \%)$ full-text articles were retrieved for review concerning $\operatorname{ADR}^{(11-18,20,24-25,28-29,34-36,38-40,46,51-}$ $52,54,56), 14(29.8 \%) \mathrm{DDI}^{(21-23,27,30-33,41,43-45,49,53)}$ and nine $(19.1 \%)$ are related to both $D D I$ and $\operatorname{ADR}^{(19,26,37,42,47-}$ 48,50,55,57), according to Figures 2 and 3.

\section{Discussion}

To the best of our knowledge, this is the first integrative review examining studies published between 2008-2013 on the occurrence of DDIs and ADRs specifically among older adults. This study helps to demonstrate that the issue in focus is a prevalent problem in various countries. However, generalizing the results of this review is difficult because of the multiplicity of methods and sample sizes of several studies and the diversity of locations they were conducted. Some aspects are highlighted.

Initially, different definitions of polypharmacy were utilized in the studies analyzed such as $\geq 3^{(33)}$, $\geq 5^{(19-20,26-27,29,31,37,42,44,46,51)}, \quad \geq 6^{(52)}$ or $\geq 10^{(11-12,15)}$. In thirty-one studies there was no definition of polypharmacy (13-14,16-18,21-25,28,30,32,34-36,38-41,43,45,47-50,53-57). Therefore, there is a need for a clear cut-off point that defines polypharmacy worldwide. A definition focusing on whether the medication is clinically indicated may be more appropriate( ${ }^{(9)}$ than the number of ingested medicines.

DDI and ADR are frequently the end result of polypharmacy as shown in the studies analyzed(11-57), and are associated with others predictors, for example: sex differences ${ }^{(24,33)}$; sex of patients, alcohol consumption and smoking habits ${ }^{(29)}$; increased age ${ }^{(39,49)}$; diagnoses of diseases and multiple comorbidities ${ }^{(21,25,48-49,53)}$; use the specific types drugs, such as patients using clopidogrel with proton pump inhibitors ${ }^{(14-15)}$, tamoxifen(18), coprescription of macrolide antibiotics and calcium-channel blockers ${ }^{(23)}$, trimethoprim/sulfamethoxazole ${ }^{(17,28,34)}$, antid epressants( ${ }^{(31,36)}$,warfarin(38,45), benzodiazepines ${ }^{(47)}$; also, cognitive impairment and various functional problems that affect practical drug management capacity(24-25); living situation ${ }^{(19)}$; access to health care, prescription of drug therapy regimens by two or more prescribers ${ }^{(49)}$; and educational status ${ }^{(31,44,50)}$.

Non-adherence to treatment is a common problem in older adults. DDIs and ADRs during hospitalization have been reported to be associated with nonadherence, which are also common among older adults who are discharged from hospital and are using several drugs for their chronic diseases. Studies examining readmissions due to DDIs and consequent ADRs were also performed ${ }^{(44,51,55)}$. Therefore, early detection and recognition of clinically important interactions by healthcare professionals are vital for monitoring the occurrence of DDIs and ADRs in the continuum of health care.

Older adults usually do better use of medicines when their care is managed by a multidisciplinary team, consisting of a physician (geriatrician), clinical pharmacist and nurse. The involvement of a dentist in this team seems to be relevant, as demonstrated in a study(32).

Inappropriate prescription, with misuse of medication, poor quality of doctors' choices of prescriptions, over-prescription of drugs, additional medicines prescribed to treat side effects, and poor team-patient relationships may increase the chances of occurrences of DDI and ADR. By combining their knowledge and skills, a comprehensive plan and dosage adjustments can be developed to enable best pharmacotherapy while the risks of DDI and ADR are reduced. An efficient communication between these professionals and coordination across multiple prescribers is crucial for success. Moreover, educational programs should be conducted to improve the habit 
of prescribing rationally. Equally, patient education at discharge and follow-up is also very important.

Use of multiple medications increases the risk of inappropriate prescribing. Different interventions to optimize prescribing appropriateness in older adults, for example, the Beers' criteria, most often used in the United States ${ }^{(12,16,20,47)}$, the validated Screening Tool of Older Persons' Potentially Inappropriate Prescriptions (STOPP) and Screening Tool to Alert doctors to the Right, i.e. appropriate, indicated Treatment (START) criteria ${ }^{(11,16,30)}$ in the Ireland and United Kingdom, the Norwegian General Practice criteria (NORGEP)(41,43), and the instrument Medication Appropriateness Index (MAI $^{(40,57)}$ have been explored in studies. The studies reviewed indicated that STOPP/START criteria identified a higher proportion of inappropriate prescription than Beers' criteria.

This review showed that there are different frequencies and types of DDIs and ADR, which are drug-related problems, associated with different classes of drugs. In daily care practice, the correct diagnosis of these problems requires skill and expertise of the multidisciplinary team, especially when older adults present themselves with nonspecific complaints and manifestations. To recognize and diagnose this undesirable outcome, goals should be set in the health care service, highlighting the role of the clinical pharmacist, who uses interventions for identification and minimization the drug-related problem (DRP), as demonstrated in studies ${ }^{(4-57)}$.

Balancing the risks and benefits of multiple drug therapies may be useful in the establishment of rational interventions for the safe use of drugs. Accordingly, the use of technologies in the monitoring of DDIs and recognition of ADRs, such as computer-based screening, could help practitioners to recognize potential and clinically significant interactions and adverse events. The software must have high sensitivity and specificity, and high positive and negative predictive value. In the same way, the advantage of utilizing computerized databases for reviewing the medications' prescriptions is evident.

Thus, the reviewed studies reinforced the notion that polypharmacy is multifactorial and is associated with negative health outcomes, as reported previously in two studies reviewed ${ }^{(8-9)}$, and in the article of experts opinion that present information specifically of 12 studies about DDI and ADR(58).

One aspect noted and that needs to be investigated further, relates to examine how self-medication with over-the-counter drugs and complementary medications contribute to increases the risk of DDIs and ADRs, hospitalization and death of older adults. Other gap noted in literature relates to the methods utilized by primary care providers when assessing polypharmacy. Additionally, little information is available about the incidence/prevalence of DDIs and ADRs among older adults in developing countries.

So, this review points out the relevance of conducting more studies to explore different aspects, considering the need to develop preventive practices to guarantee the safety of older adults with regard to DDIs and ADRs.

\section{Limitations and strength of study}

Firstly, publication bias which cannot be measured due to both language and database limitations. Selection bias may have occurred because unpublished research and/or development were not selected. Possibly, another limiting aspect is the lack of methodological assessment and risk of bias due to the heterogeneity of the reviewed studies. A major strength of this study is rigorous review of the literature, specifically on the consequences of DDIs and ADRs related to polypharmacy, with diverse methodological designs, including studies published worldwide.

\section{Conclusion and implications for advanced practice in geriatric nursing}

This study has identified that early detection and recognition of clinically important DDI and ADR by healthcare professionals are vital to identify patients who are at higher risk for such events and require more cautious pharmacotherapy management to avoid negative outcomes. Thus, the potential risk to DDI and ADR can be managed by professionals with appropriate prescriptions, monitoring, and patient education in the continuum of care of older adults, i.e. through best practices.

In this sense, the professionalization on advanced nursing practice is essential, as a requirement for acquisition of knowledge, skills training and skills for making safe and effective care decisions, for example, aimed at health care of older adults commonly exposed to polypharmacy. Thus, this integrative review can help to increase awareness and discussion, to implement universal health coverage and universal access to health care of the older adults in order to guarantee the quality of care by geriatric nurses. 


\section{References}

1. World Health Organization and US National Institute on Aging. Global Health and Aging report. [Internet]. 2011. [Acesso 28 jul 2015]. Disponível em: http:// d2cauhfh6h4x0p.cloudfront.net/s3fs-public/global_ health_and_aging.pdf.

2. Silva LRF. From old age to third age: the historical course of the identities linked to the process of ageing. Hist Cienc Saude-Manguinhos. [Internet]. 2008 [Acesso 28 jul 2015];15(1):155-68. Disponível em: $\quad$ http://www.scielo.br/scielo.php?pid=S010459702008000100009\&script=sci_arttext\&tIng=en

3. Troen BR. The biology of aging. Mt Sinai J Med. [Internet]. 2003 [Acesso 28 jul 2015];70:3-22. Disponível em: https://d2cauhfh6h4x0p.cloudfront. net/s3fs-public/biology-of-aging_1.pdf?iMmu_ oMbm0b066xNEmC5mioamo2LwmEp

4. Gurwitz JH, Field TS, Harrold LR, Rothschild J, Debellis $\mathrm{K}$, Seger AC, et al. Incidence and preventability of adverse drug events among older persons in the ambulatory setting. JAMA. [Internet]. 2003 [Acesso 28 jul 2015]; 289(9):1107-16. Disponível em: http://jama. jamanetwork.com/article. aspx?articleid =196099

5. Vonbach P, Dubied A, KrähenbühIS, Beer JH. Prevalence of drug-drug interactions at hospital entry and during stay of patients in internal medicine. Eur J Intern Med. [Internet]. 2008 [Acesso 13 mar 2016];19(6):413-20. doi: 10.1016/j.ejim.2007.12.002

6. Alomar MJ. Factors affecting the development of adverse drug reactions (Review article). Saudi Pharm J. [Internet]. 2014 [Acesso 13 mar 2016];22(2):83-94. doi: $10.1016 /$ j.jsps.2013.02.003

7. Whittemore $\mathrm{R}$, Knafl $\mathrm{K}$. The integrative review: update methodology. J Adv Nurs. [Internet]. 2005 [Acesso 13 mar 2016];52(5):546-53. Doi:10.1111/j.13652648.2005.03621.x

8. Hajjar ER, Cafiero AC, Hanlon JT. Polypharmacy in elderly patients. Am J Geriatr Pharmacother. [Internet]. 2007 [Acesso 12 mar 2016];5(4):345-51. doi: 10.1016/j.amjopharm.2007.12.002

9. Fulton MM, Allen ER. Polypharmacy in the elderly: a literature review. J Am Acad Nurse Pract. [Internet]. 2005 [Acesso 12 mar 2016];17(4):123-32. doi: 10.111/j.1041-2972.2005.0020.x

10. Ganong LH. Integrative reviews of nursing research. Res Nurs Health. [Internet]. 1987 [Acesso 12 mar 2016];10(1):1-11. doi: 10.1002/nur.4770100103

11. Gallagher P, O'Mahony. STOPP (Screening Tool of Older Persons' potentially inappropriate Prescriptions): application to acutely ill elderly patients and comparison with Beers' criteria. Age Ageing. [Internet]. 2008 [Acesso 12 mar 2016];37(6):673-9. doi: 10.1093/ ageing/afn197.

12. Gallagher PF, Barry PJ, Ryan C, Hartigan I, O'Mahony $\mathrm{D}$. Inappropriate prescribing in an acutely ill population of elderly patients as determined by Beers' Criteria. Age Ageing. [Internet]. 2008 [Acesso $12 \mathrm{mar}$ 2016];37(1):96-101. doi: 10.1093/ageing/afm116

13. Zhang M, Holman CDJ, Price SD, Sanfilippo FM, Preen DB, Bulsara MK. Comorbidity and repeat admission to hospital for adverse drug reactions in older adults: retrospective cohort study. BMJ. [Internet]. 2009 [Acesso 12 mar 2016];338:a2752. doi: http://dx.doi. org/10.1136/bmj.a2752

14. Juurlink DN, Gomes T, Ko DT, Szmitko PE, Austin $\mathrm{PC}$, Tu JV, et al. A population-based study of the drug interaction between proton pump inhibitors and clopidogrel. CMA]. [Internet]. 2009 [Acesso 12 mar 2016];180(7):713-8. doi: 10.1503/cmaj.082001

15. Rassen JA, Choudhry NK, Avorn J, Schneeweiss S. Cardiovascular outcomes and mortality in patients using clopidogrel with proton pump inhibitors after percutaneous coronary intervention or acute coronary syndrome. Circulation. [Internet]. 2009; 120:2322-9. doi: 10.1161/CIRCULATIONAHA.109.873497

16. Miquel MDC, Cuervo MS, Silveira ED, Machuca IS, González-Blazquez S, Errasquin BM, et al. Potentially inappropriate drug prescription in older subjects across health care settings. Eur Geriat Med. [Internet]. 2010 [Acesso 13 mar 2016];1(1):9-14. doi:10.1016/j. eurger.2009.12.002

17. Weir MA, Juurlink DN, Gomes T, Mamdani M, Hackam DG, Jain AK, et al. Beta-blockers, trimethoprimsulfamethoxazole, and the risk of hyperkalemia requiring hospitalization in the elderly: a nested casecontrol study. Clin J Am Soc Nephrol. [Internet]. 2010 [Acesso 13 mar 2016];5(9):1544-51. doi: 10.2215/ CJN.01970310.

18. Kelly CM, Juurlink DN, Gomes T, Duong-Hua M, Pritchard KI, Austin PC, et al. Selective serotonin reuptake inhibitors and breast cancer mortality in women receiving tamoxifen: a population based cohort study. BMJ. [Internet]. 2010; [Acesso 15 mar 2016];340:c693. doi: $10.1136 / \mathrm{bmj} . c 693$.

19. Somers M, Rose E, Simmonds D, Whitelaw C, Calver J, Beer C. Quality use of medicines in residential aged care. Aust Fam Physician. [Internet]. 2010 [Acesso 16 mar 2016]; 39(6):413-6. Available from: http:// 
www.racgp.org.au/download/documents/AFP/2010/ June/201006somers.pdf

20. Harugeri A, Joseph J, Parthasarathi G, Ramesh M, Guido S. Potentially inappropriate medication use in elderly patients: a study of prevalence and predictors in two teaching hospitals. J Postgrad Med. [Internet]. 2010 [Acesso 13 mar 2016];56(3):186-91. doi: 10.4103/0022-3859.68642.

21. Bacic-Vrca V, Marusic S, Erdeljic V, Falamic S, GojoTomic N, Rahelic D. The incidence of potential drug-drug interactions in elderly patients with arterial hypertension. Pharm World Sci. [Internet]. 2010 [Acesso 13 mar 2016];32(6):815-21. doi: 10.1007/s11096-010-9442-5. 22. Rahmawati F, Hidayati N, Rochmah W, Sulaiman SAS. Potentiality of drug-drug interactions in hospitalized geriatric patients in a private hospital, Yogyakarta, Indonesia. Asian J Pharm Clin Res. [Internet]. 2010 [Acesso 13 mar 2016];3(3):191-4. Available from: http://www.academia.edu/3579826/POTENTIALITY_ OF_DRUG-DRUG_INTERACTIONS_IN_HOSPITALIZED_ GERIATRIC_PATIENTS_IN_A_PRIVATE_HOSPITAL_ YOGYAKARTA_INDONESIA

23. Wright AJ, Gomes T, Mamdani MM, Horn JR, Juurlink DN. The risk of hypotension following coprescription of macrolide antibiotics and calciumchannel blockers. CMA]. [Internet]. 2011 [Acesso 11 mar 2016];183(3):303-7. doi: 10.1503/cmaj.100702

24. Steinman MA, Lund BC, Miao Y, Boscardin WJ, Kaboli PJ. Geriatric conditions, medication use, and risk of adverse drug events in a predominantly male, older veteran population. Am Geriatr Soc. 2011 [Acesso 15 mar 2016];59(4):615-21. doi: 10.1111/j.15325415.2011.03331.x

25. Steinman MA, Hanlon JT, Sloane RJ, Boscardin WJ, Schmader KE. Do geriatric conditions increase risk of adverse drug reactions in ambulatory elders? Results from the VA GEM drug study. J Gerontol A Biol Sci Med Sci. [Internet]. 2011 [Acesso 17 mar 2016];66(4):44451. doi: $10.1093 /$ gerona/glq236

26. Santos RM, Sette IMF, Belém LF. Drug use by elderly inpatients of a philanthropic hospital. Braz J Pharm Sci. [Internet]. 2011 [Acesso 17 mar 2016];47(2):391-2. dx.doi.org/10.1590/S1984-82502011000200021

27. Mendes-Netto RS, Silva CQV, Oliveira AD Filho, Rocha $C E$, Lyra-Junior DP. Assessment of drug interactions in elderly patients of a family health care unit in Aracaju (Brazil): a pilot study. Afr J Pharm Pharmacol. [Internet]. 2011;5:812-8. DOI: 10.5897/AJPP10.299

28. Antoniou T, Gomes T, Mamdani MM, Yao Z, Hellings

C, Garg $A X$, et al. Trimethoprim-sulfamethoxazole induced hyperkalaemia in elderly patients receiving spironolactone: nested case-control study. BMJ. [Internet]. 2011 [Acesso 17 mar 2016];343:d5228. doi: http://dx.doi.org/10.1136/bmj.d5228

29. Varallo FR, Capucho HC, Planeta CS, Mastroianni PC. Safety assessment of potentially inappropriate medications (PIM) use in older people and the factors associated with hospital admission. J Pharm Pharmaceut Sci. [Internet] 2011 [Acesso 20 mar 2016];14(2):28390. Disponível em: https://ejournals.library.ualberta.ca/ index.php/JPPS/article/view/9795/8379

30. Gallagher PF, O'Connor MN, O'Mahony D. Prevention of potentially inappropriate prescribing for elderly patients: a randomized controlled trial using STOPP/ START criteria. Clin Pharmacol Ther. [Internet]. 2011 [Acesso 20 mar 2016];14(2):283-90. doi: 10.1038/ clpt.2011.44

31. Hanlon JT, Wang $X$, Castle NG, Stone RA, Handler SM, Semla TP, et al. Potential underuse, overuse and inappropriate use of antidepressants in older veteran nursing home patients. J Am Geriatr Soc. [Internet]. 2011 [Acesso 13 mar 2016];59(8):1412-20. doi: 10.1111/j.1532-5415.2011.03522.x.

32. Skaar DD, O'Connor H. Potentially serious drugdrug interactions among community-dwelling older adult dental patients. Oral Surg Oral Med Oral Pathol Oral Radiol Endod. [Internet]. 2011 [Acesso 13 mar 2016];112(2):153-60. doi: 10.1016/j. tripleo.2011.03.048

33. Venturini CD, Engroff $P$, Ely LS, Zago LFA, Schroester G, Gomes I, et al. Gender diferences, polypharmacy, and potential pharmacological interactions in the elderly. Clinics. (São Paulo). [Internet]. 2011 [Acesso 12 mar 2016];66(11):1867-72. DOI: 10.1590/S180759322011001100004

34. Antoniou T, Gomes T, Mamdani MM, Juurlink DN. Trimethoprim/sulfamethoxazole-induced phenytoin toxicity in the elderly: a population-based study. $\mathrm{Br} \mathrm{J}$ Clin Pharmacol. [Internet]. 2011;71(4):544-9. doi: 10.1111/j.1365-2125.2010.03866.x.

35. Budnitz DS, Lovegrove MC, Lovegrove N, Richards CL. Emergency hospitalizations for adverse drug events in older americans. N Engl J Med. [Internet]. 2011 [Acesso 12 mar 2016];365:2002-12. DOI: 10.1056/ NEJMsa1103053

36. Coupland C, Dhiman P, Morriss R, Arthur A, Barton G, Hippisley-Cox J. Antidepressant use and risk of adverse outcomes in older people: population based cohort study. BM]. [Internet]. 2011 [Acesso 12 mar 2016];343:d4551. doi: 10.1136/bmj.d4551. 
37. Chan DC, Chen JH, Kuo HK, We CJ, Lu IS, Chiu LS, et al. Drug-related problems (DRPs) identified from geriatric medication safety review clinics. Arch Gerontol Geriatr. [Internet]. 2012 [Acesso 12 mar 2016];54(1):168-74. doi: 10.1016/j.archger.2011.02.005

38. Baillargeon J, Holmes HM, Lin YL, Raji MA, Sharma G, Kuo YF. Concurrent use of warfarin and antibiotics and the risk of bleeding in older adults. Am J Med. [Internet]. 2012 [Acesso 12 mar 2016];125(2):183-9. doi: 10.1016/j.amjmed.2011.08.014.

39. O'Connor MN, Gallagher P, Byrne S, O'Mahony D. Adverse drug reactions in older patients during hospitalization: are they predictable? Age Ageing. [Internet]. 2012 [Acesso 12 mar 2016];41(6):771-6. doi: $10.1093 /$ ageing/afs046.

40. Somers A, Mallet L, Cammen TVA, Robays H, Petrovic M. Applicability of an adapted medication appropriateness index for detection of drug-related problems in geriatric inpatients. Am J Geriatr Pharmacother. [Internet]. 2012 [Acesso 12 mar 2016];10(2):101-9. doi: 10.1016/j. amjopharm.2012.01.003

41. Bakken MS, Ranhoff AH, Engeland A, Ruths S. Inappropriate prescribing for older people admitted to an intermediate-care nursing home unit and hospital wards. Scand J Prim Health Care. [Internet]. 2012 [Acesso 12 mar 2016];30(3):169-75. doi: 10.3109/02813432.2012.704813.

42. Romana A, Kamath L, Sarda A, Muraraiah S, CR Jayanthi. Polypharmacy leading to adverse drug reactions in elderly in a tertiary care hospital. Int J Pharm Bio Sci. [Internet]. 2012 [Access 12 mar 2016];3(3):21824. Available from: http://www.ijpbs.net/vol-3/issue-3/ pharma/23.pdf

43. Halvorsen $\mathrm{KH}$, Granas AG, Engeland A, Ruths S. Prescribing quality for older people in Norwegian nursing homes and home nursing services using multidose dispensed drugs. Pharmacoepidemiol Drug Saf. [Internet]. 2012 [Access 12 mar 2016];21(9):929-36. doi: 10.1002/pds.2232.

44. Mehuys E, Dupond L, Petrovic M, Christiaens T, Van Bortel $L$, Adriaens $E$, et al. Medication management among home-dwelling older patients with chronic diseases: possible roles for community pharmacists. J Nutr Health Aging. [Internet]. 2012 [Access 12 mar 2016];16(8):721-6. doi: 10.1007/s12603-012-0028-x. 45. Pincus D, Gomes T, Hellings C, Zheng H, Paterson JM, Mamdani MM, et al. A population-based assessment of the drug interaction between levothyroxine and warfarin.
Clin Pharmacol Ther. [Internet]. 2012 [Access 12 mar 2016];92(6):766-70. doi: 10.1038/clpt.2012.171.

46. Marcum ZA, Amuan ME, Hanlon JT, Aspinall SL, Handler SM, Ruby CM, et al. Prevalence of unplanned hospitalizations caused by adverse drug reactions in older veterans. J Am Geriatr Soc. [Internet]. 2012;60(1):3441. doi: 10.1111/j.1532-5415.2011.03772.x

47. Vila JV, Vila MMV, Ibáñez LS, Zaragoza JAA, Royo LM. Adecuación de la utilización de benzodiazepinas en ancianos desde la oficina de farmacia. Un estudio de colaboración médico-farmacéutico. Aten Primaria. [Internet]. 2012 [Access 12 mar 2016];44(7):402-10. doi:10.1016/j.aprim.2011.07.018

48. Obreli-Neto PR, Nobili A, Lyra-Júnior DP, Pilger D, Guidoni $C M$, Baldoni $A O$, et al. Incidence and predictors of adverse drug reactions caused by drug-drug interactions in elderly outpatients: a prospective cohort study. J Pharm Pharmaceut Sci. [Internet]. 2012[Access 12 mar 2016];15(2):332-43. Available from: https:// ejournals.library.ualberta.ca/index.php/JPPS/article/ view/17049/13684

49. Obreli-Neto PR, Nobili A, Marusic S, Pilger D, Guidoni $\mathrm{CM}$, Baldoni $\mathrm{AO}$, et al. Prevalence and predictors of potential drug-drug interactions in the elderly: a crosssectional study in the Brazilian primary public health system. J Pharm Pharmaceut Sci. [Internet]. 2012 [Access 11 mar 2016];15(2):344-54. Available from: https://ejournals.library.ualberta.ca/index.php/JPPS/ article/viewFile/12209/13688

50. Ramanath KV, Nedumballi S. Assessment of medication-related problems in geriatric patients of a rural tertiary care hospital. J Young Pharm. [Internet]. 2012 [Access 11 mar 2016];4(4):273-8. doi: 10.4103/0975-1483.104372

51. Bonnet-Zamponi D, d'Arailh L, Konrat C, Delpierre $S$, Lieberherr $D$, Lemaire $A$, et al. Drug-related readmissions to medical units of older adults discharged from acute geriatric units: results of the optimization of medication in AGEd multicenter randomized controlled trial. J Am Geriatr Soc. [Internet]. 2013 [Access 11 mar 2016];61(1):113-21. doi: 10.1111/jgs.12037

52. Nickel $\mathrm{CH}$, Ruedinger JM, Messmer AS, Maile $\mathrm{S}$, Peng $A$, Bodmer $M$, et al. Drug-related emergency department visits by elderly patients presenting with non-specific complaints. Scand J Trauma Resusc Emerg Med. [Internet]. 2013 [Access 11 mar 2016];21:15. doi: 10.1186/1757-7241-21-15.

53. Marusic S, Bacic-Vrca V, Obreli-Neto PR, Franic M, Erdeljic V, Gojo-Tomic N. Actual drug-drug interactions in 
elderly patients discharged from internal medicine clinic: a prospective observational study. Eur J Clin Pharmacol. [Internet]. 2013 [Access 11 mar 2016];69(9):1717-24. doi: 10.1007/s00228-013-1531-7.

54. Bondesson A, Eriksson T, Kraig A, Holmdahl L, Midlöv $P$, Höglund P. In-hospital medication reviews reduce unidentified drug-related problems. Eur J Clin Pharmacol. [Internet]. 2013 [Access 11 mar 2016];69(3):647-55. doi: $10.1007 / \mathrm{s} 00228-012-1368-5$.

55. Yeoh TT, Si P, Chew L. The impact of medication therapy management in older oncology patients. Support Care Cancer. [Internet]. 2013 [Access 11 mar 2016];21(5):1287-93. doi: 10.1007/s00520-0121661-y.

56. Klopotowska JE, Wierenga PC, Smorenburg SM, Stuijt CCM, Arisz L, Kuks PFM, et al. Recognition of adverse drug events in older hospitalized medical patients. Eur ] Clin Pharmacol. [Internet]. 2013 [Access 11 mar 2016];69(1):75-85. doi: 10.1007/s00228-012$1316-4$

57. Somers A, Robays H, De Paepe P, Van Maele G, Perehudoff K, Petrovic M. Evaluation of clinical pharmacist recommendations in the geriatric ward of a Belgium university hospital. Clin Interv Aging. [Internet]. 2013 [Access 11 mar 2016];8:703-9. doi: 10.2147/CIA.S42162.

58. Maher RL, Hanlon J, Hajjar ER. Clinical consequences of polypharmacy in elderly. Expert Opin Drug Saf. [Internet]. 2014 [Access 11 mar 2016];13(1):57-65. doi: $10.1517 / 14740338.2013 .827660$
Copyright @ 2016 Revista Latino-Americana de Enfermagem This is an Open Access article distributed under the terms of the Creative Commons (CC BY).

This license lets others distribute, remix, tweak, and build upon your work, even commercially, as long as they credit you for the original creation. This is the most accommodating of licenses offered. Recommended for maximum dissemination and use of licensed materials. 